\title{
Estimation of Seismic Vulnerability Levels of Urban Structures With Multisensor Remote Sensing
}

\author{
Christian Geiß, Member, IEEE, Marianne Jilge, Tobia Lakes, and Hannes Taubenböck
}

\begin{abstract}
The ongoing global transformation of human habitats from rural villages to ever growing urban agglomerations induces unprecedented seismic risks in earthquake prone regions. To mitigate affiliated perils requires the seismic assessment of built environments. Numerous studies emphasize that remote sensing can play a valuable role in supporting the extraction of relevant features for preevent vulnerability analysis. However, the majority of approaches operate on building level. This induces the deployment of very high spatial resolution remote sensing data, which hampers, nowadays, utilization capabilities for larger areas due to data costs and processing requirements. In this paper, we alter the spatial scale of analysis and propose concepts and methods to estimate the seismic vulnerability level of homogeneous urban structures. A procedure is designed, which comprises four main steps dedicated to: 1) delineation of urban structures by means of a tailored unsupervised data segmentation procedure with scale optimization; 2) characterization of urban structures by a joint exploitation of multisensor data; 3 ) selection of most feasible features under consideration of in situ vulnerability information; and 4) estimation of seismic vulnerability levels of urban structures within a supervised learning framework. We render the prediction problem in three ways to address operational requirements that can evolve in real-life situations. 1) To discriminate two or more classes based on labeled samples of all classes present in the data under investigation, we use the framework of soft margin support vector machines $(C$-SVM). 2) To consider situations, where solely labeled samples are available for the class(es) of interest and not for all classes present in the data, we deploy ensembles of $\nu$-one-class SVM ( $\nu$-OC-SVM). and 3) To fit data with a higher statistical level of measurement (interval or ratio scale), we utilize a support vector regression (SVR) approach to estimate a regression function from the training samples. Experimental results are obtained for the earthquake-prone mega city Istanbul, Turkey. We use multispectral data from the RapidEye constellation, elevation measurements from the TanDEM-X mission, and spatiotemporal analyses based on data from the Landsat archive to characterize the urban environment. In addition, different in situ data sets are incorporated for Istanbul's district Zeytinburnu and the residual
\end{abstract}

Manuscript received January 20, 2015; revised May 05, 2015; accepted May 27, 2015. This work was supported in part by the European Commission's Seventh Framework Programme (FP7/2007-2013) under Grant 312972 "Framework to integrate Space-based and in situ sENSing for dynamic vUlnerability and recover Monitoring," and in part by Helmholtz-EOS (Earth Observation System).

C. Geiß is with the German Aerospace Center (DLR), German Remote Sensing Data Center (DFD), 82234 Oberpfaffenhofen-Weßling, Germany, and also with the Department of Geography, Humboldt Universität zu Berlin, 12489 Berlin, Germany (e-mail: christian.geiss@dlr.de).

M. Jilge and H. Taubenböck are with the German Aerospace Center (DLR), German Remote Sensing Data Center (DFD), 82234 OberpfaffenhofenWeßling, Germany (e-mail: marianne.jilge@dlr.de; hannes.taubenboeck@ dlr.de).

T. Lakes is with the Department of Geography, Humboldt Universität zu Berlin, 12489 Berlin, Germany (e-mail: tobia.lakes@geo.hu-berlin.de).

Color versions of one or more of the figures in this paper are available online at http://ieeexplore.iee.org.

Digital Object Identifier 10.1109/JSTARS.2015.2442584 settlement area of Istanbul. When estimating damage grades for Zeytinburnu with SVR, best models are characterized by mean absolute percentage errors less than $11 \%$, and fairly strong goodness of fit $(R>0.75)$. When aiming to identify different types of urban structures for the remaining settlement area of Istanbul (i.e., urban structures determined by large industrial/commercial buildings and tall detached residential buildings, which can be considered here as highly and slightly vulnerable, respectively), results obtained with $C$-SVM show a distinctive increase of accuracy compared to results obtained with ensembles of $\nu$-OC-SVM. The latter were not able to exceed moderate agreements, with $\kappa$ statistics slightly above 0.45 . Instead, $C$-SVM allowed obtaining $\kappa$ statistics expressing substantial and even excellent agreements ( $\kappa>0.6$ up to $\kappa>0.8$ ). Overall, analyzes provide very promising empirical evidence, which confirms the potential of remote sensing to support seismic vulnerability assessment.

Index Terms-Earthquakes, Istanbul, Landsat, machine learning, object-based image analysis, RapidEye, seismic vulnerability assessment, support vector machines (SVM), TanDEM-X.

\section{INTRODUCTION}

$\mathbf{T}$ HE IMPACT of natural hazards such as earthquakes on mankind has increased dramatically over the last decades. Global urbanization processes and increasing spatial concentration of exposed elements, such as people, buildings, infrastructure, and economic values in earthquake prone regions induce seismic risk at a uniquely high level. Thereby, the increase of urban population has occurred during a time period that is comparatively short with respect to the return time of severe earthquakes. Earthquakes that had little impact in the past, when they hit sparsely populated and spatially fragmented settlement areas, will nowadays shake urban agglomerations with millions of people. This situation, when left unmitigated, is expected to cause unprecedented death tolls, enormous economic and ecological losses, critical infrastructure and service failures, and poses a significant threat for civil security, and a sustainable development in the future [1]-[3]. The mitigation of affiliated perils requires detailed knowledge about seismic risks. As an important constituent element of seismic risk, the seismic vulnerability of the built environment needs to be assessed. In particular, it is crucial to have information about the building inventory and its behavior with respect to a certain level of ground shaking [4].

Conventional approaches to assess the seismic vulnerability of the building inventory, which incorporate an exclusive application of detailed in situ building-by-building analysis by structural engineers, may provide very detailed and highquality vulnerability information, but are decreasingly able to cope with the high spatiotemporal dynamics of urban environments. On the contrary, information collected on a 
very broad spatial level, such as spatially aggregated census data hampers the consideration of small-scale hazard effects in a downstream risk model [5]. Hence, building inventory data and affiliated seismic vulnerability information is often outdated, spatially aggregated and discontinuous, and in many earthquake prone regions of the world simply not existent.

Remote sensing has already proven its great potential to extract relevant features for preevent vulnerability analysis of buildings. The intrinsic advantage of remote sensing is the ability to offer an overview of building stocks and serve as a screening method for derivation of building vulnerability related features, such as shape characteristics, height, roof material, period of construction, structure type, and spatial context [6]. In this sense, the authors of [7] and [8] present a proxy-based seismic vulnerability assessment approach primarily using the features "period of construction" and "building height" for an area-wide assessment. Generally, this topic is subject to a lively research and has gained much scientific contemplation in the past few years. Thereby, the research environment is constituted by two distinctive science communities: remote sensing and earthquake engineering. Nowadays, concepts and methods have reached a level, where they are found to be relevant and being accepted in both communities. This is evident since the following studies are published in established journals and publication series of both communities.

The authors of [9]-[11] characterize the built environment by means of remote sensing data and retrieve specific fragility functions or damage probability matrices, respectively, for designated building types. The authors of [12] assess the vulnerability of buildings in a hybrid way, by combining in situ ground truth for selected buildings with information derived from remote sensing data. Supervised classification techniques are subsequently applied to classify the residual building inventory. The authors of [13] and [14] derive basic building parameters from remote sensing (i.e., "roof type" and "building height") and assess the seismic vulnerability of buildings in conjunction with in situ data based on an expert judgment method and categorization according to the European Macroseismic Scale (EMS-98) using supervised regression and classification techniques, respectively. More exhaustively, the authors of [15] and [16] combine detailed in situ seismic vulnerability information with a huge number of features derived from multisensor remote sensing data describing the urban morphology. Supervised regression and classification techniques are then applied to evaluate the suitability for an area-wide seismic vulnerability assessment and earthquake loss estimation. All cited studies present promising empirical evidence with respect to the viability of the approaches. However, from a conceptual point of view, all approaches operate on building level. This induces the deployment of very high spatial resolution (VHR) remote sensing data, which hampers utilization capabilities for larger areas due to data costs and processing requirements. When aiming at spatially continuous and consistent assessment approaches that are applicable for large areas, those kinds of data represent, nowadays, a clear limitation.
To alleviate these restrictions, analyses can be addressed at a coarser level of the urban morphology. From a conceptual point of view, urban environments are perceived as systems that exhibit a hierarchical arrangement [17]. At the lowest level of aggregation, individual objects such as buildings can be considered, followed by their homogeneous assemblage at block level (i.e., urban structures), and end with a spatially indifferent urban area at the highest level of aggregation. In this paper, we alter the spatial scale of analysis compared to the aforementioned studies and propose concepts and methods to estimate vulnerability levels for physically homogeneous urban structures. Thus, a coarser level of urban morphology is addressed when compared to building level. However, this allows relying on remote sensing data with a lower spatial resolution but larger spatial coverage.

This idea goes along with the concept of urban structure types (USTs). USTs represent distinct and homogeneous spatial entities in terms of physical arrangement of their constituent elements, such as surface materials, affiliated environmental characteristics (e.g., microclimate), or functional properties, such as land use [18], [19]. From a remote sensing perspective, the concept of USTs has been exploited within different applications. Previous studies emphasize the viability of this concept to, e.g., identify distinctive settlement types [20]-[22], classify urban biotopes [23], support urban (micro-)climate modeling [24], [25], or monitor urban dynamics [17]. As can be seen from the cited studies, definitions of target class(es) to be mapped and characterized are very heterogeneous. This renders "USTs" rather a concept, then an accepted typology with defined specification. Hence, we use the term urban structures to generally describe distinctive and homogeneous assemblages of land cover/land use elements.

In the context of seismic vulnerability research, the authors of [26] note already that urban structures can be helpful to assess the seismic vulnerability of buildings, since they represent a characteristic grouping of idealtypic building types and the spatial context they are embedded in. In this manner, the authors of [5] and [27] use multitemporal Landsat data to discriminate homogeneous urban structures based on an image segmentation approach and semantically annotate them by utilizing a supervised classification scheme. Derived urban structures are intended to serve as strata for a guided and more detailed analysis of the building stock with VHR optical data and a ground-based omnidirectional imaging system. The sensed information is combined with ancillary information (i.e., information from the world housing encyclopedia) for a subsequent probabilistic seismic vulnerability assessment. Hence, the approach delineates and determines urban structures without prior vulnerability knowledge and assesses the seismic vulnerability a posteriori.

In contrast to that, here, we present a procedure, which learns available prior seismic vulnerability knowledge for urban structures, which are characterized exclusively based upon spaceborne remote sensing systems. Such a procedure can be considered, in particular, relevant in situation where an in situ assessment has been conducted, but, for instance, only for spatially fragmented and discontinuous settlement areas, and the gathering of VHR data and additional ground-based 


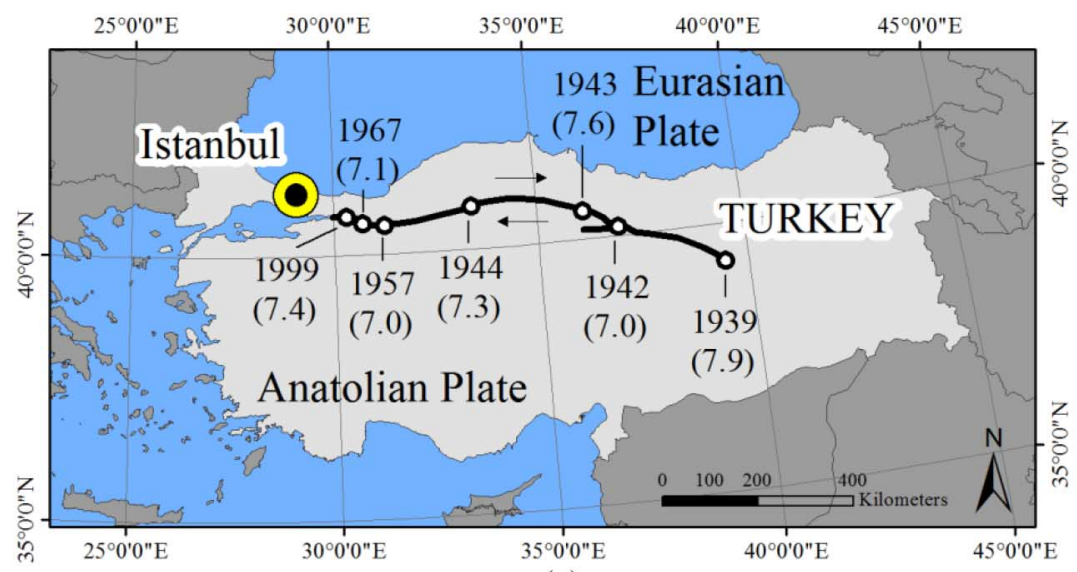

(a)

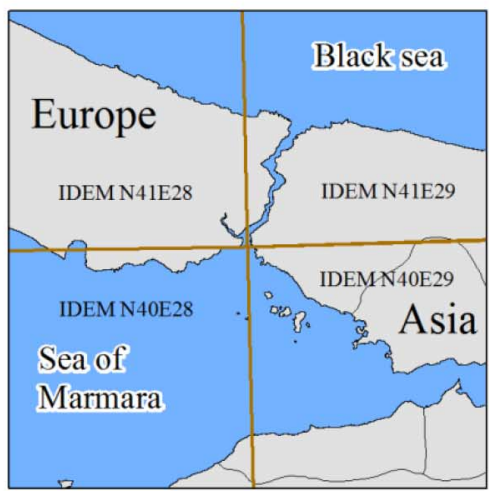

(c)

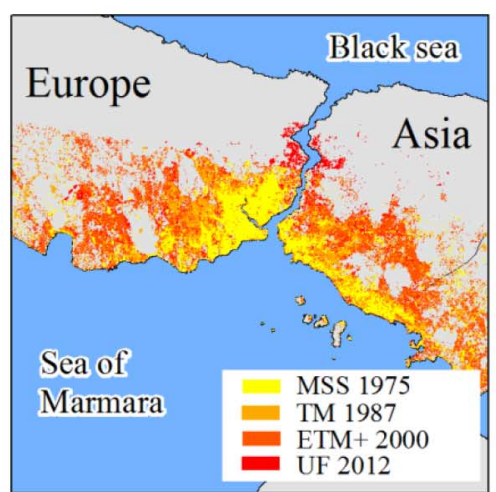

(d)

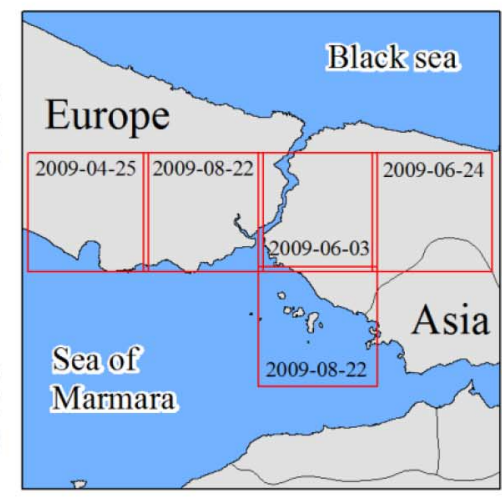

(b)

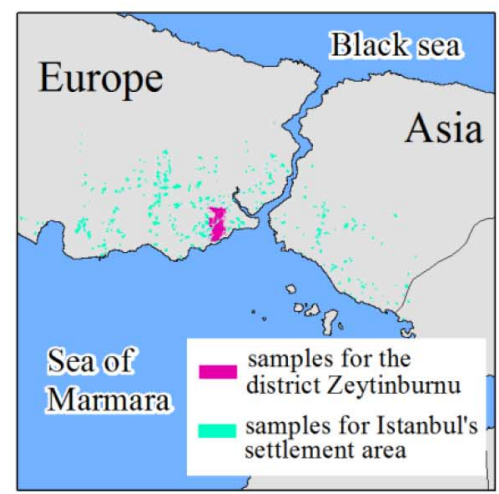

(e)

Fig. 1. Overview on the location of the study area, tectonic setting and acquired data. (a) Location of Istanbul and main ruptures along the North Anatolian Fault since 1939 (moment magnitude of affiliated earthquake in brackets), which indicate a westbound regime. (b) Acquired RapidEye data. (c) Tiles of TDM intermediate DSM data. (d) Spatiotemporal evolvement of urban structures in Istanbul monitored with data from the Landsat archive (MSS 1975, TM 1987, and ETM+ 2000) and TDM (UF 2012). (e) In situ information for the district Zeytinburnu and for Istanbul's settlement area.

information is not feasible. Thus, the primal objective of this paper is to introduce an original procedure for estimation of seismic vulnerability levels of urban structures with earth observation data. Thereby, we evaluate, in this rather new thematic application context, if desired target variables can be estimated at all with the selected remote sensing data and designed procedures. In particular, we combine multispectral data and elevation measurements to characterize the urban morphology and use techniques of object-based image analysis [28], [29] and statistical learning [30], [31] to estimate the vulnerability level of urban structures under consideration of in situ information. Thereby, a perceptual coherence [32] of seismic vulnerability properties and physical appearance of urban structures as well as the composition of their constituent elements, as measured with remote sensing, are assumed. As such, in this paper, we follow the general principle to infer and examine an empirical relation between in situ data and multisensor remote sensing for estimation of seismic vulnerability levels.

A number of remote sensing systems appear to be promising for the characterization of urban structures. In this paper, we exploit multispectral and elevation data, which feature a lower spatial resolution compared to systems with the highest spatial resolution available nowadays (e.g., QuickBird-2, Geoeye-1, WorldView-2, etc.), but allow at the same time a larger spatial coverage. In particular, we use multispectral data from the RapidEye constellation [33], elevation measurements from the TanDEM-X mission (TDM) [34], and spatiotemporal analyses generated from data from the Landsat archive [35]. Based on the remote sensing data and subsequently derived information layers, a procedure is designed, which comprises four main steps dedicated to: 1) delineation of urban structures by means of tailored unsupervised data segmentation procedure with scale optimization; 2) characterization of urban structures using multisensor data; 3) selection of most feasible features under consideration of in situ vulnerability information; and 4) estimation of seismic vulnerability levels of urban structures within a supervised learning framework.

Three challenging experiments are designed, which are intended to demonstrate feasibility of procedures and discuss viability of results. Thereby, we relate our experimental analyses to Istanbul, Turkey, since the city faces an enormous seismic threat [4]. In addition, in situ observations are available for relevant parts of the city. This paper is organized as follows. Section II gives an overview on study area, utilized remote sensing data and reference information. Section III presents developed and deployed methods. We use Section IV to give an overview on the concepts and objectives of designed experiments and report and discuss outcomes of 


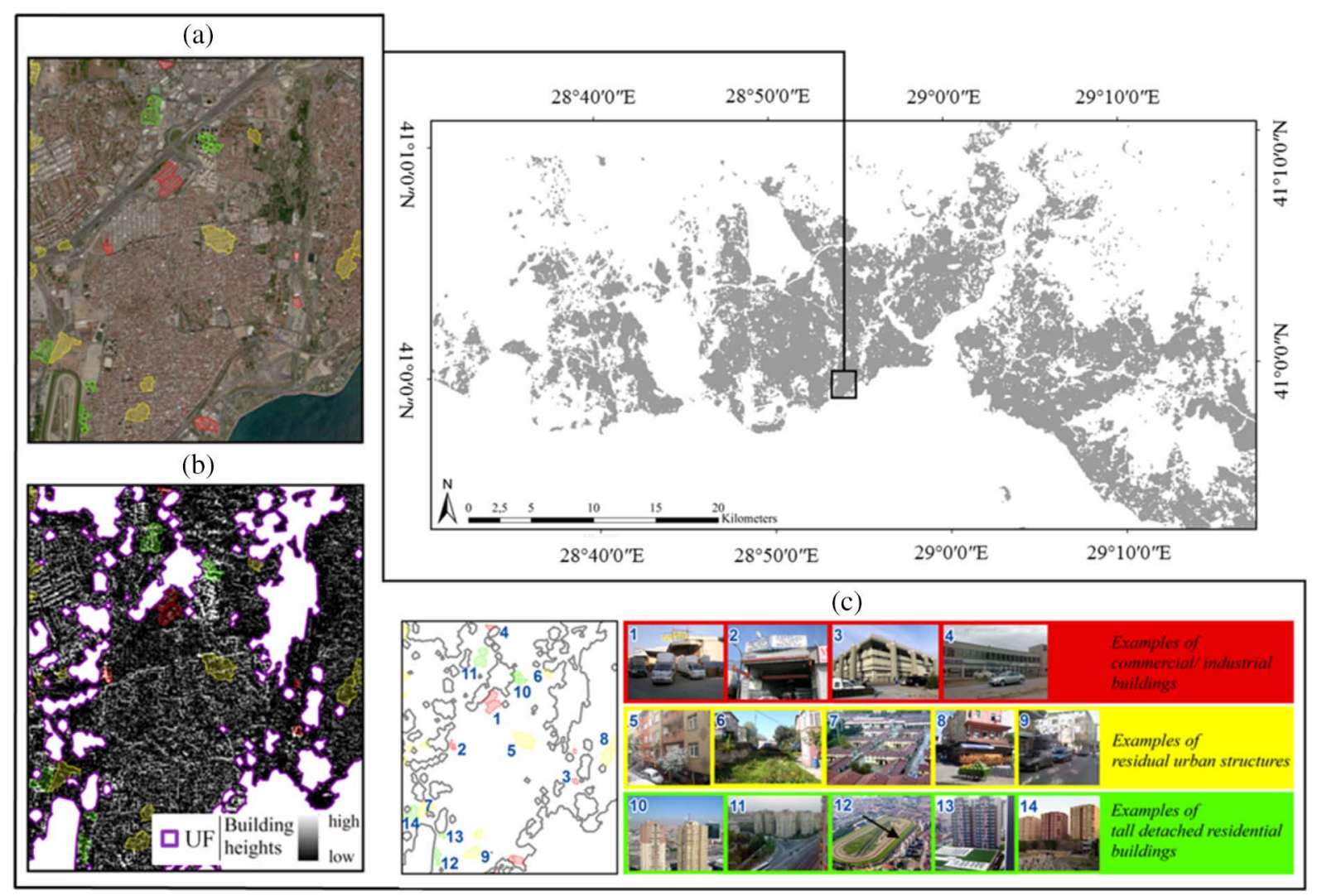

Fig. 2. (a) Exemplifications of RapidEye data and (b) nDSM TDM data with affiliated appearance of urban structures. (c) Images from Google Panoramio, which were deployed for identification of urban structures according to relevant types.

experiments in Section V. Concluding remarks and some future perspectives are given in Section VI.

\section{Study AREA AND DATA}

\section{A. Istanbul, Turkey}

Istanbul $\left(41^{\circ} 1^{\prime} \mathrm{N}, 28^{\circ} 58^{\prime} \mathrm{O}\right)$ has about 14.2 million inhabitants [36], which constitutes it a mega city. Some sources estimate the number of people living in Istanbul to be even higher, since numerous people are not registered and, thus, do not appear in official statistics. Massive migration during past decades induced a complex and erratic city structure, which comprises small and low-rise informal settlements (depicted as Gecekondular in Turkish), large industrial buildings, and highrise residential and commercial buildings, among others. About one-fifth of the existing buildings were built within the past 15 years [37]. Besides, many buildings were expanded by new stories and extensions without permission. Moreover, authorized real estate projects were already modified during the construction phase. It is estimated that the majority of buildings in Istanbul does not correspond to any structural standards and lack regulatory supervision. Hence, it is assumed that most of the buildings are not shake resistant, although a law to enforce resistant design was issued in 1999.

Almost all earthquakes in Turkey are related to tectonic movement [38]. Istanbul is located close to the North Anatolian Fault [NAF; Fig. 1(a)]. The NAF represents an active rightlateral strike-slip fault. Previous earthquakes along the fault indicate a westbound regime [39] and give rise to a possible earthquake close to Istanbul. According to the authors of [40], a 35-70\% probability for the occurrence of an earthquake with a magnitude $>7$ along the NAF within the next 30 years is expected.

\section{B. Remote Sensing Data: RapidEye, TDM, and Landsat}

For the assessment of urban structures with respect to their seismic vulnerability level, we select a multisensor approach, comprising three different kinds of earth observation systems, to extract complementary information. The RapidEye constellation was chosen, since it appears to be promising for area-wide analyses of urban structures. It offers a high spatial resolution of $6.5 \mathrm{~m}$, in conjunction with a swath width of $77 \mathrm{~km}$, and a maximum acquisition capacity of $1500 \mathrm{~km}$ per orbit ([41]; please note that also missions such as Sentinel-2 [42] with a spatial resolution of $10 \mathrm{~m}$ can be considered as an interesting opportunity with respect to a suitable trade-off of spatial resolution and swath width). It consists of five equally designed satellites, which operate in a single sun-synchronous orbit. We acquired five RapidEye images, which were collected between April and August 2009. They cover the main parts of the settlement area of Istanbul [Figs. 1(b) and 2(a)]. Cloud coverage was minimal and imagery was delivered at level 2A. Hence, data were subject to geometric and radiometric sensor corrections and were calibrated to at-sensor radiances with a pixel size of $6.5 \mathrm{~m}$ at nadir. In the experiments, we 
deploy the blue $(0.440-0.510 \mu \mathrm{m})$, green $(0.520-0.590 \mu \mathrm{m})$, red $(0.630-0.685 \mu \mathrm{m})$, and nir $(0.760-0.850 \mu \mathrm{m})$ band, and neglect RapidEye's specific red edge band $(0.690-0.730 \mu \mathrm{m})$. We want to rely on image bands that are frequently available for multispectral scanners and thus render methods and results more transferable and ubiquitous (although, e.g., Sentinel-2, or Landsat-8 [43], can be considered as an interesting option to acquire additional spectral information).

Second, elevation measurements from the TDM were chosen to characterize height properties of urban structures for large areas. The TDM is a spaceborne radar interferometer, which acquires data for a seamless global digital surface model (DSM) with an unprecedented, globally consistent spatial resolution of 0.4 arcseconds [34] (which corresponds to a spatial resolution of $12 \mathrm{~m}$ in Istanbul). This allows resolving objects that are elevated from the earth's surface in urban environments, such as buildings. In our study, four tiles of the so-called TDM Intermediate Digital Elevation Model (IDEM) are used [Tile N40E028, N40E029, N41E028, and N41E029; Figs. 1(c) and 2(b)]. The IDEM consists only of the best quality singlebaseline processed data of the first global coverage, whereas insufficient acquisitions affected by phase-unwrapping errors are excluded.

Finally, data from the Landsat archive, which dates back to 1972 , are considered, since they enable a characterization of spatiotemporal developments of urban structures (Fig. 1(d); [44]). Data were acquired by the multispectral scanner (MSS), thematic mapper (TM) sensor, and the enhanced thematic mapper (ETM+) sensor in 1975, 1987, and July 2000, respectively. Images are constituted by four (MSS) and seven (TM, ETM+) multispectral bands covering a spectral range of $0.500-1.100 \mu \mathrm{m}$ (MSS) and 0.450-2.350 $\mu \mathrm{m}$ (TM, ETM+) at $79 \mathrm{~m}$ and $30 \mathrm{~m}$ spatial resolution, respectively.

\section{In Situ Data: Seismic Building Vulnerability Reference Information}

Two different in situ data sets are incorporated for Istanbul's district Zeytinburnu and the settlement area of Istanbul [Fig. 1(e)]. As such, it should be noted that the experiments elaborated later in Section IV-A are based on different kinds of in situ data.

1) For Istanbul's district Zeytinburnu $\left(40^{\circ} 59^{\prime} \mathrm{N}, 28^{\circ} 54^{\prime} \mathrm{O}\right)$, a spatially continuous and complete assessment of the buildings according to the capacity spectrum method [45] was available. The method consists of three main steps. 1) Quantification of the seismological impact in terms of a response spectrum; thereby, possible seismic events are considered, which results in a function showing displacement in dependency of acceleration. 2) The capacity of individual structures is quantified in dependency of specific strength and deformation properties of their components. 3) Finally, the capacity of an individual structure (in the form of a pushover curve) is related to the seismological impact on the structure (in the form of response spectra), whereas the intersection of the two functions approximates the response of the structure. Thus, it allows quantifying expectable damage grades in a probabilistic manner. The necessary in situ information to adapt the functions for Zeytinburnu was gathered and provided by the authors of [9] ([9] provide also are more detailed explanation of the method and its adaption).

2) In addition, ground-based GPS-photos for large parts of the settlement area were available from Google Panoramio [some exemplifications for relevant structures are depicted in Fig. 2(c)]. Generally, such information can support a rapid visual screening assessment of buildings [46]. Here, the information was used to identify urban structures according to distinctive types as identified by the capacity spectrum method. In particular, we identified urban structures determined by large industrial/ commercial buildings or tall detached residential buildings, which can be considered as highly and slightly vulnerable in this application, respectively. Find a more detailed description of procedures in Section IV-A, where we describe the experiments.

\section{METHODS}

Presented data are subject to a multistep procedure to assess seismic vulnerability of urban structures. A block scheme of the procedure is provided in Fig. 3. First, remote sensing data are preprocessed. The multispectral data are atmospherically corrected by means of the software tool ATCOR-2 [47], and a mosaic is generated from the different images (Section III-A). Data from the TDM serve for both the derivation of a settlement mask (i.e., urban footprint, UF; Section III-A1), and the computation of a normalized digital surface model (nDSM; Section III-A2). Landsat imagery are deployed for spatiotemporal analyses, and are subject to a post classification change detection procedure (Section III-A3). Subsequently, four main steps are carried out within a supervised learning framework. 1) Section III-B is used to describe an unsupervised image segmentation procedure with scales optimization to allow for a delineation of urban structures. 2) Section III-C details the calculations of multiscale features from both the multispectral data and height information from the $\mathrm{nDSM}$ for a comprehensive characterization of urban structures. 3) Section III-D features are grouped according to different underlying remote sensing data and segmentation scales to better understand the respective value within the process of supervised learning; in addition, supervised feature selection techniques are deployed to possibly identify the most suitable feature sets for model learning; this is naturally done under consideration of in situ data, which determine the UST with affiliated vulnerability level. 4) Finally, in Section III-E, we briefly reveal three different formulations of support vector machines (SVM), for multi- and one-class problems, and function estimation within a supervised learning framework, which were used in the experiments.

\section{A. Preprocessing}

Multispectral RapidEye data were subject to atmospheric corrections with ATCOR-2 [47] to account for radiometric distortions. The five images were radiometrically adjusted with a histogram match and merged to a spatially continuous mosaic. 


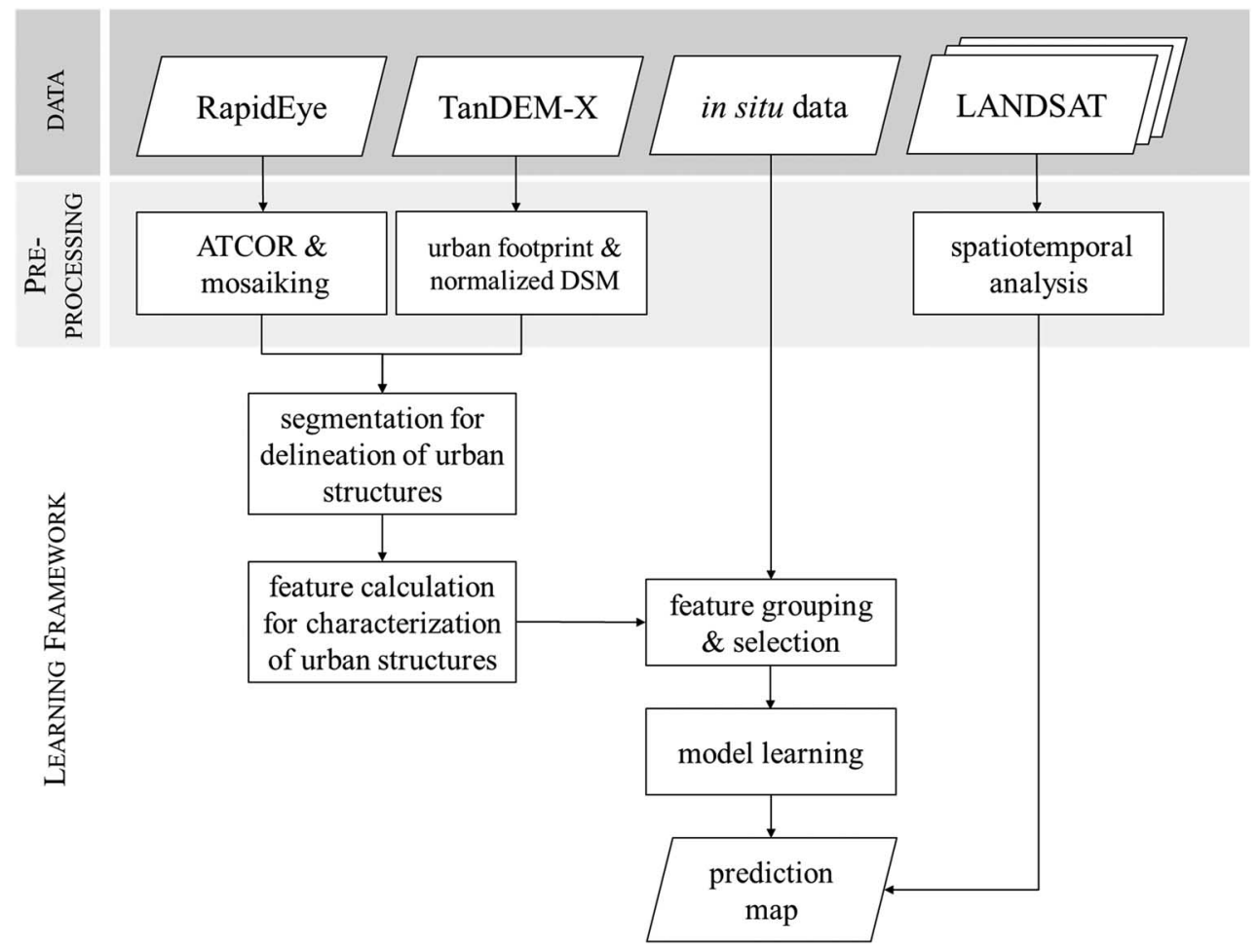

Fig. 3. Block scheme of the proposed procedure.

RapidEye and TDM imagery were resampled (by means of a nearest neighbor interpolation) and coregistered to a common spatial resolution of $5 \mathrm{~m}$.

1) Derivation of Settlement Mask From TDM Data: To focus analyses on urban environments, we deploy a fully automated image analysis procedure to discriminate "built-up" and "nonbuilt-up" land cover (referred to as "UF"; [48]). Based on the TDM, which collects two global data sets of very high resolution synthetic aperture radar images between 2011 and 2013, built-up areas can be delineated globally with an unknown spatial detail. The binary layer is generated with an unsupervised classification scheme accounting for both the original backscattering amplitude and extracted texture characteristics. A high classification accuracy, which exceeds consistently an overall accuracy of $94 \%$ and a $\kappa$ statistic of 0.75 for representative case studies [48], ensures a reliable delineation of built-up areas. The original UF data were subject to some generalization procedures in this study. We deployed morphological operators (i.e., closing was performed by sequentially carry out dilation and erosion [49]) to ensure a spatially nonfragmented representation of the settlement area of Istanbul. The utilized UF of Istanbul covers an area of more than $500 \mathrm{~km}^{2}$ and is visualized in Fig. 1(d).

2) Calculation of Normalized DSM From TDM: Height characteristics are frequently used features for seismic vulnerability assessment [6], [15], [27]. We use elevation measurements of TDM IDEM to compute a nDSM, which comprises elevation information of objects above ground. To this purpose, a digital terrain model (DTM) is derived from the data first with a region growing-based progressive morphological filter procedure [50]. This approach was recently proposed to address general challenges associated with the use of morphological filters in nonflat terrain, and overcomes individual challenges related to the spatial resolution of TDM data. It comprises a multistep procedure using concepts of morphological image filtering, region growing, and interpolation techniques. It is based on the idea of progressive morphological filters that aim to discriminate ground and nonground pixels in the DSM based on algebraic set operations. Some free parameters need to be determined when using this approach. To ensure extraction of all buildings present in the area under investigation, the structuring element must always exceed a building's outline. The side length of the structuring element was determined empirically for eight individual subsets of the study area. In addition, an elevation difference threshold and similarity constraint need to be fixed. Both were set in accordance to previous experimental analyses, to ensure a favorable trade-off between decrease of omission errors and increase of commission errors when classifying ground pixels (i.e., $2.6 \mathrm{~m}$ for elevation difference threshold and $0.8 \mathrm{~m}$ for similarity constraint; [50]). Identified ground pixels are interpolated to a DTM based on inverse distance weighting [51]. Finally, the DTM was subtracted from the DSM to receive the final $\mathrm{nDSM}$.

3) Spatiotemporal Analysis With LANDSAT: To uncover the spatiotemporal evolvement of urban structures in Istanbul, we integrate results from a post classification change detection procedure provided by the authors of [44]. Based on LANDSAT imagery, the settlement area of Istanbul is identified [Fig. 1(d)]. The approach deploys spectral classification in conjunction with a temporal hierarchical scheme. This means that the classified settlement area for a past time step is used as a spatial condition when classifying urbanized areas for the more current 
time steps. The individual classifications feature viable accuracies. The overall accuracy, as evaluated based on comparison with VHR data [44], is $93.7 \%$ for the year $1975(\kappa=0.82)$, $92.4 \%(\kappa=0.79)$ for the year 1987 , and $90.8 \%(\kappa=0.82)$ for 2000, respectively.

Data from the Landsat archive are used quite frequently to estimate the period of construction of built environments in seismic vulnerability studies (e.g., [12], [5]). The period of construction is a feature that might encode valuable information with respect to seismic vulnerability properties of urban structures due to, e.g., the implementation of building codes or usage of distinctive and idiosyncratic construction materials and techniques associated with certain construction periods. From a conceptual perspective, this feature is different from the features introduced in Section III-C with respect to both thematic information content and statistical level of measurement. That is why, we assign the estimated period of construction posterior to model learning [see Fig. 3 in this section and Fig. 9 in Section V] to further discriminate urban structures that might exhibit similar physical properties, as measured with the RapidEye, and TDM data, but belong to different categories of construction periods. Simultaneously, this reduces the computational burden compared to an assignment prior to model learning, since then a model must be learned based on labeled samples for every class of interest and different periods of construction.

\section{B. Segmentation Procedure for Delineation of Urban Structures}

This section is dedicated to the description of an unsupervised segmentation procedure with scale optimization for delineation of urban structures. In contrast to natural environments, urban man-made structures have been identified as few examples of objects within a landscape with distinct and crisp boundaries, which feature often also an irregular shape [52]. This makes the utilization of object-based image analysis techniques feasible and especially the latter limits the use of uniform spatial entities, such as quadratic objects. Image segmentation represents the basis for object-based image analysis and aims at the delineation of meaningful real-world objects [29]. In this study, segmentation focuses on the distinction of intra-urban areas, which are homogeneous in terms of their response in the multispectral and surface elevation data. For a joint exploitation, a brightness layer was defined as the sum in the visible, NIR, and elevation measurement bands $z_{i(v)}$ divided by the number of affiliated bands $\left(n_{v}\right)$

$$
B=\frac{1}{n_{v}} \sum_{i=1}^{n_{v}} z_{i(v)} .
$$

In this study, segments represent basic spatial units to delineate and characterize homogeneous urban structures later on. We deploy multiresolution segmentation based on fractal net evolution approach (FNEA) [53]. This is a bottom-up regiongrowing segmentation algorithm. It starts from individual pixels and merges pixels in dependence of user-defined constraints related to spectral and geometrical properties of modeled segments. Based on this technique, several automated routines are

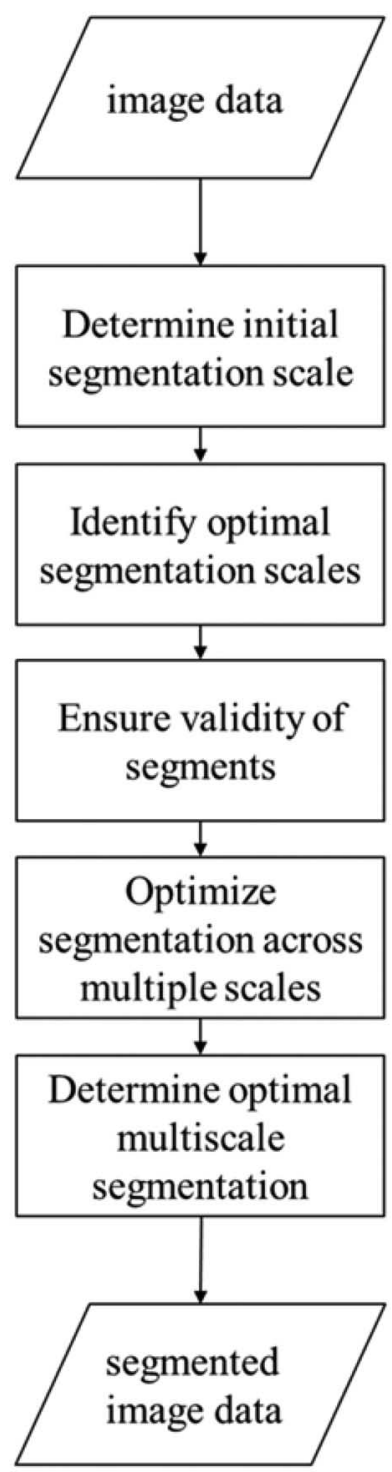

Fig. 4. Schematic processing steps of the segmentation procedure.

carried out in a sequential way to ensure parameterization and outcomes of optimized multiresolution image segmentation to be objective, data-driven, and generic. A schematic overview of the segmentation procedure is given in Fig. 4.

First, an initial image level is built, which includes all "builtup" areas from the UF data set [Section III-A1; exemplifications are provided in Appendix A, Fig. 12(a)]. The subsequent segmentation is limited to areas identified as "built-up". The multiresolution segmentation approach is controlled by scale, color, and shape. Thereby, shape is being composed of compactness and smoothness. The scale parameter is a function of the geometric resolution of the image data and defines the maximum allowable heterogeneity of modeled segments, with a lower scale parameter resulting in a higher number of segments. We suggest to put more emphasize on shape heterogeneity rather than on grey-value heterogeneity. This is due to the fact that man-made features, such as urban structures, have distinct shape and size properties, unlike, e.g., natural features. Analogously, we propose to maintain the same weights for 
heterogeneity of smoothness and compactness throughout the segmentation. However, the main difficulty lays in the determination of a scale, which is suitable to represent different kinds of urban structures adequately. In this manner, the usage of a single scale is prohibited, since urban structures feature several orders of magnitude of spatial extend. Thus, we adapt a multilevel segmentation procedure proposed by the authors of [54], which compares the distinctiveness of segments generated at multiple scales. Prior to this, we deploy the objective function introduced by the authors of [55] to identify scene-specific optimal segmentation scales. Based on the assumption that optimal segmentation maximizes intrasegment homogeneity and intersegment heterogeneity, a measure is calculated by incorporating intrasegment variance and Moran's $I$.

During the procedure, we suggest to maintain constant weights for color and shape criteria and create segments at ascending scales in a defined interval, e.g., $\left[h_{s}, h_{s+1}, \ldots\right.$, $\left.h_{n}\right]$. Thereby, $h_{s}$ represents the initial scale. To determine an appropriate initial scale and ensure that generated segments can represent valid delineations of urban structures, some constraints are introduced. In this sense, we define that valid image segments must correspond to the Shannon sampling theorem. This theorem states that modeled segments should be of the order of one-tenth of the dimension of the sampling scheme, the pixel, to ensure that they will be completely independent of their random position and their orientation in relation to the sampling scheme [29]. Thus, we define that there should be at least ten pixels to represent a valid segment in terms of size, and five pixels to represent a valid segment in terms of width

$$
\text { valid segment }= \begin{cases}1, & \text { if } \frac{\sqrt{A}}{c} \cdot \frac{1}{10}>1 \wedge S<3 \\ 1, & \text { if } \frac{d}{c} \cdot \frac{1}{5}>1 \wedge S \geq 3 \\ 0, & \text { else }\end{cases}
$$

where $A$ is the area of a segment, $c$ represents the pixel size of the image data, $d$ is the width of a segment, and $S$ a shape complexity index calculated as perimeter to boundary ratio

$$
S=\frac{q}{2 \cdot r \cdot \pi}, \quad r=\sqrt{\frac{A}{\pi}}
$$

where $q$ is the perimeter of a segment, and $r$ is the radius of circle with the same surface area. Analogous to the author of [56], we chose a threshold of 3 to differentiate between compact $(S<3)$ and narrow/long $(S \geq 3)$ segments. Generally, a suitable $h_{s}$ should include a comparatively small share of nonvalid segments (nvSs) and represent a local maximum regarding the decline in shares of nvSs. This principle and the selection of $h_{s}$ for our application are illustrated in Section IV-B [Fig. 7(a)].

Following the aforementioned heuristic [55], for each of the created scales in the given interval, the intrasegment variance $\left(\sigma^{2}\right)$ with respect to the brightness values was calculated

$$
\sigma^{2}=\frac{\sum_{i=1}^{n} A_{i} \sigma_{i}^{2}}{\sum_{i=1}^{n} A_{i}}
$$

where $A_{i}$ and $\sigma_{i}^{2}$ represent the area and intrasegment variance of segment $i$. The intrasegment variance $\sigma^{2}$ is the weighted average, with the areas of each segment being the weights. As a measure of intersegment heterogeneity, Moran's $I$ is used

$$
I=\frac{N}{\sum_{i} \sum_{j} e_{i j}} \cdot \frac{\sum_{i} \sum_{j} e_{i j}\left(\mu B_{i}-\mu \bar{B}\right)\left(\mu B_{j}-\mu \bar{B}\right)}{\sum_{i}\left(\mu B_{i}-\mu \bar{B}\right)^{2}}
$$

where $N$ is the number of segments indexed by $i$ and $j ; \mu B$ is the mean brightness of a segment; $\mu \bar{B}$ is the mean brightness of all segments; and $e_{i j}$ is the spatial weight between segments $i$ and $j$,implemented in our case analogous to a queen contiguity or 8-connectivity as follows:

$$
e_{i j}= \begin{cases}1, & \text { if } i, j \text { are adjacent neighbor segments } \\ 0, & \text { else. }\end{cases}
$$

With the determined variance and autocorrelation measure, the objective function is calculated by summing up normalized values of $\sigma^{2}$ and $I$

$$
F\left(\sigma^{2}, I\right)=\frac{\sigma_{\max }^{2}-\sigma^{2}}{\sigma_{\max }^{2}-\sigma_{\min }^{2}}+\frac{I_{\max }-I}{I_{\max }-I_{\min }} .
$$

Generally, the objective function is calculated for ascending scales. Thereby, $\sigma^{2}$ values represent a monotonic function: for the sequel $\left(\sigma^{2}{ }_{n}\right)_{\mathrm{n} \in \mathbb{R}}$, the condition $\sigma_{n+1}^{2} \geq \sigma_{n}^{2}$ is fulfilled. The maximum value of function $F\left(\sigma^{2}, I\right)$ is a statistical indicator of optimal segmentation [Section IV-B, black arrow in Fig. 7(b)]. As discussed above, a single optimal scale is not sufficient to take into account the relationship between the spatial structure of an image and the structure of urban environments. Thus, we deploy a plateau objective function $F(u)$, which was introduced by the authors of [57], to obtain multiple optimal scales. The function is defined as

$$
F(u)=F\left(\sigma^{2}, I\right)_{\max }-\sigma
$$

where $F\left(\sigma^{2}, I\right)_{\max }$ represents the maximum value of the objective function, and $\sigma$ is the standard deviation of the function calculated for all scales. Optimal segmentation scales should be located above the plateau function, since these scales have high external and low internal heterogeneity levels, and the balance between under- and oversegmentation is still present [57]. As initial segmentation scale for the subsequent multilevel optimization procedure, we select the segmentation of the first scale above the plateau objective function [Section IV-B, black arrow in Fig. 7(b); exemplified in Appendix A, Fig. 12(b), ioMRS].

1) Optimization Procedure: Segments that do not represent valid segments according to the Shannon sampling theorem criteria defined above [exemplified in Appendix A, Fig. 12(c-I), nvS] are merged with adjacent neighbor segments that have the smallest mean brightness difference of all adjacent neighbor segments [Fig. 12(c-II), ioMRS-m]. Subsequently, a second hierarchical scale level is created and the mean percentage difference $(m P D)$ between subsegment level $(L 1)$ and supersegment level $(L 2)$ is calculated as

$$
m P D=\frac{\left|\mu B_{L 1}-\mu B_{L 2}\right|}{\mu B_{L 2}}
$$


where $\mu B$ is the mean brightness of the respective super- and subsegments. Similar to the simplified and data-driven version of the multiscale optimization approach proposed by the authors of [58], we regard segments as "real" subsegment if their $m P D$ exceeds the mean $m P D$ of all subsegments by more than two standard deviations

$$
\text { real subsegment }= \begin{cases}1, & m P D>2 \sigma_{m P D} \\ 0, & \text { else. }\end{cases}
$$

Identified "real" subsegments [exemplified in Appendix A, Fig. 12(d-I), rSS] are transferred to the supersegment level [Fig. 12(d-II)]. Then, the similarity of transferred adjacent subsegment is evaluated in terms of their mean brightness values, and two adjacent segments are merged [Fig. 12(d-III)] if they fulfill the following condition:

$$
\begin{aligned}
& \text { similarity }_{\text {segment } 1, \text { segment } 2} \\
& \quad= \begin{cases}1, & \left|\mu B_{\text {segment } 1}-\mu B_{\text {segment } 2}\right| \leq \gamma \\
0, & \text { else }\end{cases}
\end{aligned}
$$

with $\gamma$ being a threshold. The procedure is repeated for the remaining scales above the plateau objective function, whereby the result of the previous cycle becomes the subsegment level in each step, and a number of segments are merged to create a supersegment level above, according to the respective scale factor [58]. The complete procedure is intended to provide an optimized segmentation that comprises distinctive and valid segments independent of their particular scale. However, as the multilevel optimization procedure and the merging of nvSs alter image segments and alignment, we calculated the objective function again for the processed levels to identify the most sufficient final segmentation [fS, Section IV-B, black arrow in Fig. 7(c); exemplified in Appendix A, Fig. 12(e), fS].

\section{Feature Calculation for Characterization of Urban Structures}

Features based on the RapidEye and TDM nDSM data were computed to allow for a comprehensive characterization of urban structures. We extracted statistical measures of first and second order from both the multispectral RapidEye data and TDM nDSM (Fig. 5). Measures of central tendency and measures of spread of the different image bands are deployed. The same measures are computed for the nDSM to take into account height characteristics of discriminated areas. Additionally, rotation-invariant texture measures for the optical and nDSM data were computed based on the co-occurrence matrix (GLCM; [59]). It could be shown that texture can provide valuable information when aiming at discrimination of urban structures [52], and generally allow to overcome a lack of spectral information [60]. The last group of features consists of spatial metrics. Using the NDVI [61], we quantify the share of vegetation per segment. Thereby, pixels, which exceed a certain NDVI value (i.e., 0.3), are considered as vegetation. To specifically describe height characteristics of elevated objects that do not represent vegetation within a segment (i.e., buildings), we first identify pixels that exceed a certain nDSM value (i.e., $2.6 \mathrm{~m}$ ). Besides the share of elevated objects per segment, measures of central tendency and spread of elevated objects are computed.

We calculated the aforementioned features not only for the optimized multiscale segmentation, which generally provides the spatial entities for subsequent procedures, but also for two segmentations with a larger scale factor (i.e., scale factor 80 and 120), which were not subject to the optimization procedure. This was done to ensure a sufficient consideration of spatial context information of urban structures. In fact, it is very challenging to fully exclude oversegmentation by means of an unsupervised segmentation procedure. When urban structures are subject to oversegmentation, a characteristic appearance with respect to spectral-spatial composition may not be reflected. However, including information from coarser segmentation scales allows to model spatial context relations in feature space adequately. This idea is consistent with approaches that deploy supersegment information to enhance classification accuracy (e.g., [62] and [63]).

Overall, each segment of the optimized multiscale segmentation is represented by a 198-dimensional feature vector, containing 66 different features computed for three segmentation levels.

\section{Feature Grouping and Selection}

Two different strategies were followed to bundle features into feature sets to be used in the experiments. First, a thematic grouping was carried out to quantify the usability of the different remote sensing data sets and evaluates the value of features from multiple segmentation levels. Beside the unreduced feature vector containing all computed features, we grouped all optical features, all height-related features, all optical features from the optimized segmentation level, all height-related features from the optimized segmentation level, and both all optical- and height-related features from the optimized segmentation level.

The second strategy to bundle features is motivated by the circumstance that features vectors with a high dimensionality often exhibit redundancy, are highly correlated, and may suffer from the "Hughes phenomenon," which states that for a limited amount of samples, the predictive accuracy decreases as the dimensionality of the feature vector increases [64]. Accordingly, we applied two machine learning-based feature selection algorithms on the data. The two approaches represent filter methods, which work independently with respect to the classifier, which is in contrast to the concept of wrapper methods. They were chosen since they can handle both regression problems and evaluate discrete valued variables. We used the Relief-F approach [65], because it enables to rank individual features, and deployed the correlation-based feature selection (CFS) method [66], since it enables the scoring of the value of groups of features. The applied filter methods run supervised and aim to identify the best features for building robust regression and classification models.

The Relief-F approach ranks features according to the assumption that useful features should be able to differentiate 


\section{FEATURES}

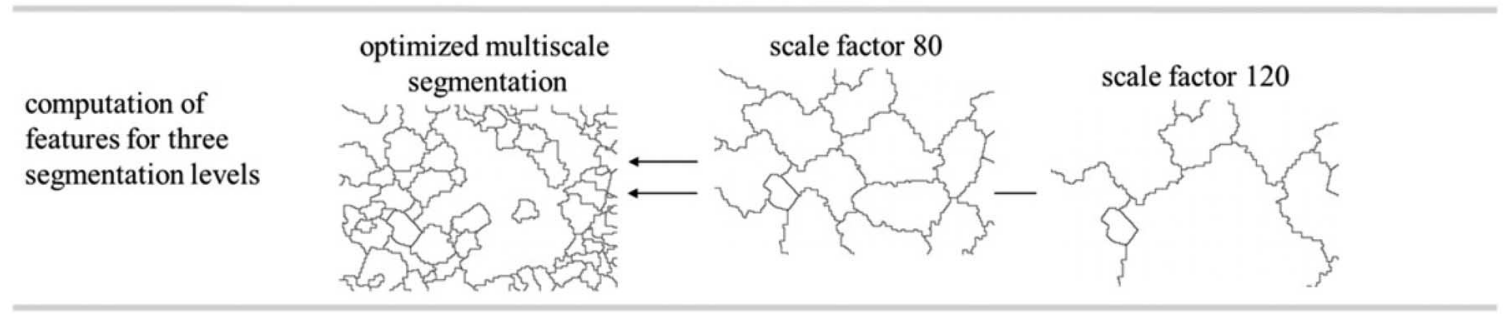

\begin{tabular}{|c|c|c|c|}
\hline & & RapidEye & TanDEM-X \\
\hline \multirow{2}{*}{ 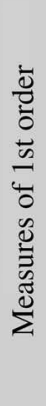 } & 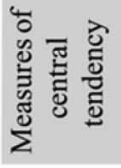 & $\begin{array}{l}\text { Mean }(\rho \mathrm{R}, \rho \mathrm{G}, \rho \mathrm{B}, \rho \mathrm{NIR}, \rho \mathrm{Brightness}, \rho \text { Max. Diff. }) \\
\text { Median }(\rho \mathrm{R}, \rho \mathrm{G}, \rho \mathrm{B}, \rho \mathrm{NIR})\end{array}$ & $\begin{array}{l}\text { Mean (nDSM) } \\
\text { Median (nDSM) }\end{array}$ \\
\hline & 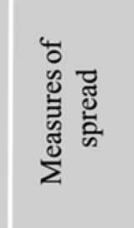 & $\begin{array}{l}\text { Standard deviation }(\rho \mathrm{R}, \rho \mathrm{G}, \rho \mathrm{B}, \rho \mathrm{NIR}) \\
\text { Variance }(\rho \mathrm{R}, \rho \mathrm{G}, \rho \mathrm{B}, \rho \mathrm{NIR}) \\
\text { Minimum }(\rho \mathrm{R}, \rho \mathrm{G}, \rho \mathrm{B}, \rho \mathrm{NIR}) \\
\text { Maximum }(\rho \mathrm{R}, \rho \mathrm{G}, \rho \mathrm{B}, \rho \mathrm{NIR}) \\
\text { Range }(\rho \mathrm{R}, \rho \mathrm{G}, \rho \mathrm{B}, \rho \mathrm{NIR}) \\
\text { Interquartile range }(\rho \mathrm{R}, \rho \mathrm{G}, \rho \mathrm{B}, \rho \mathrm{NIR})\end{array}$ & $\begin{array}{l}\text { Standard deviation (nDSM) } \\
\text { Variance (nDSM) } \\
\text { Minimum (nDSM) } \\
\text { Maximum (nDSM) } \\
\text { Range (nDSM) } \\
\text { Interquartile range (nDSM) }\end{array}$ \\
\hline 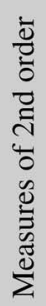 & 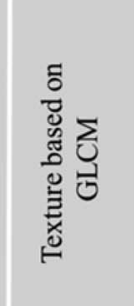 & $\begin{array}{l}\text { Homogeneity }(\rho \text { Brightness) } \\
\text { Contrast ( } \rho \text { Brightness) } \\
\text { Dissimilarity }(\rho \text { Brightness) } \\
\text { Entropy ( } \rho \text { Brightness) } \\
\text { Angular } 2^{\text {nd }} \text { moment ( } \rho \text { Brightness) } \\
\text { Mean ( } \rho \text { Brightness) } \\
\text { Standard deviation ( } \rho \text { Brightness) } \\
\text { Correlation ( } \rho \text { Brightness) }\end{array}$ & $\begin{array}{l}\text { Homogeneity }(n \mathrm{DSM}) \\
\text { Contrast ( } n \text { DSM }) \\
\text { Dissimilarity }(n \mathrm{DSM}) \\
\text { Entropy }(n \mathrm{DSM}) \\
\left.\text { Angular } 2^{\text {nd }} \text { moment ( } n \mathrm{DSM}\right) \\
\text { Mean }(n \mathrm{DSM}) \\
\text { Standard deviation ( } n \mathrm{DSM}) \\
\text { Correlation }(n \mathrm{DSM})\end{array}$ \\
\hline & 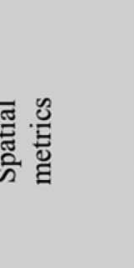 & Share of vegetation & $\begin{array}{l}\text { Share of elevated objects } \\
\text { Mean height of elevated objects } \\
\text { Standard deviation of height of elevated objects } \\
\text { Variance of height of elevated objects } \\
\text { Minimum height of elevated objects } \\
\text { Maximum height of elevated objects } \\
\text { Range of height of elevated objects }\end{array}$ \\
\hline
\end{tabular}

Fig. 5. Features derived from remote sensing data for different segmentation scales to characterize the urban morphology.

between instances from different classes and have similar values for instances from the same class [65], [67]. Therefore, an instance from the data is randomly sampled and $k$ nearest neighbors from the same and opposite classes are located. Feature values of the $k$ nearest neighbors are compared to the sampled instance and are used to update relevant scores for each feature. This procedure is repeated until a number of instances $m$ were considered. Thereby, $m$ is a free parameter, but frequently sets to the maximum number of labeled instances available to achieve a reliable approximation [65].

CFS deploys a best first search algorithm to identify a group of subsets, which are possibly suitable. The identified subsets are evaluated by means of an entropy-based heuristic. The heuristic favors subsets with a high-feature-class correlation and low-feature-feature intercorrelation. Relief-F and CFS are both multivariate procedures, which evaluate features in dependence of other features in the data set.

\section{E. Support Vector Machines}

SVM emanate from the field of machine leaning and represent a family of nonparametric approaches for supervised classification and regression [68]. They are based on the structural risk minimization principle, which suggest a trade-off between the accuracy of an approximation and the complexity of the affiliated approximation function. SVM determine a suitable set of parameters to fit a decision surface, the so-called hyperplane, between different classes of labeled samples. To deal with nonlinear problems, the labeled samples are mapped through a nonlinear transformation $\phi(\cdot)$ from the input space $\chi$ into a space of higher dimensionality $\mathcal{H}$ [Appendix B, Fig. 13(a)-(b)]. In that space, the optimal separating hyperplane maximizes the margin between the patterns of the different classes and the hyperplane [Fig. 13(c)]. The maximized margin can be described by two additional marginal hyperplanes that border the samples closest to the separating surface, the 
so-called support vectors [69], [70]. Only those samples are needed to define the model, which allows for building robust models with a high generalization capability based on a comparatively small number of labeled training samples. A linear separation in $\mathcal{H}$ corresponds to a nonlinear separation in the original input space $\chi$ [71] [Fig. 13(d)].

Different formulations of SVM allow rendering a prediction problem in three ways. 1) If two or more classes are to be discriminated based on labeled samples of all classes present in the data under investigation, the framework of $C$-SVM can be utilized. 2) If solely labeled samples are available for the class(es) of interest and not for all classes present in the data, (ensembles of) one-class $\nu$-SVM ( $\nu$-OC-SVM) can be deployed. 3 ) If the statistical level of measurement is higher and corresponds to an interval or ratio scale, a support vector regression (SVR) approach allows to estimate a prediction function from the training samples.

These three SVM formulations are revealed in Appendix B with affiliated minimization objective and decision function to clarify methodological divergences, which are relevant for differing real-world situations. Those may evolve when aiming to assess urban structures according to different engineeringrelated methods and a varying amount of prior knowledge available. For a more detailed theoretical and applicationoriented background of SVM, the reader can refer to the authors of [68], [69], [72]-[75]. In the context of remote sensing, the authors of, e.g., [76]-[80], provide comprehensive literature.

\section{DESCRIPTION OF EXPERIMENTS AND EXPERIMENTAL SETUP}

\section{A. Description of Experiments}

Experiments are designed with three different formulations of the supervised prediction problem. We render the prediction problem in three ways to address operational requirements that may evolve in many real-life cases.

1) For the district Zeytinburnu, we first aggregate the damage grades, as determined with the capacity spectrum method, from building level to structure level (generated with the multiscale segmentation procedure). In particular, the aggregated damages grades on structure level are calculated from the damage grades of the corresponding buildings, whereby a weighted average was computed taking the building areas into account. Subsequently, the aim is to estimate the damage grade for spatial entities, where no damage grade information is available based on SVR approach [Fig. 6(a)].

2) Then, we use the ground-based GPS-photos from the whole settlement area of Istanbul to identify systematically urban structures that are either determined by large industrial/commercial buildings or tall detached residential buildings. Analogous to the previous setting, the structure level provides, hereby, the constituting spatial units. Generally, the predominant building type can be seen as proxy for the vulnerability of an area [5]. In this study, those two structure types revealed distinctive properties with respect to the capacity spectrum assessment. Thereby, urban structures determined by large industrial/commercial buildings can be considered as highly vulnerable in our application, whereas urban structures determined by tall detached residential buildings can be considered as slightly vulnerable. In the second experiment, we aim to identify those two urban structures within the settlement area of Istanbul, disregarding all other potentially present classes in the area. Thereby, only labeled samples are available for the classes of interest and not for all classes present in the data [Fig. 6(b)]. This is a very challenging but realistic task. The availability of information comprising all urban structures present in the area of interest is infrequently satisfied in real-life situations. We deploy an ensemble of $\nu$-OC-SVM to address this problem.

3) However, in experiment 3 , we investigate feasibility of procedures when labeled samples of other classes are also available [Fig. 6(c)]. This renders the classification problem fully supervised and we approach it with $C$-SVM.

\section{B. Experimental Setup}

Regarding free parameters of multiresolution segmentation, we put more emphasize on shape heterogeneity (shape: $0.7)$ rather than on grey-value heterogeneity $(0.5)$ as suggested in Section III-B. First, an initial scale $h_{s}$ was determined. Therefore, the ratio of valid segments and nvSs, as evaluated with (2), was computed. Fig. 7(a) shows the corresponding function. We chose scale 24, since it features a small share of nvSs and the decline in shares of nvSs with respect to neighboring scales is comparatively explicit. Hence, the objective function is calculated for the interval $[24,25, \ldots, 150]$, and the outcome is plotted in Fig. 7(b). It can be seen that optimal segmentation scales above the plateau objective function $(F(u)=1.432)$ are located between 24 and 38 . Hence, those scales were subject to the optimization procedure described in Section III-B2 ( $\gamma$ in similarity constraint (11) was set to 5). Outcomes are reassessed with the objective function to determine the fS [black arrow in Fig. 7(c)].

Regarding the feature selection algorithms, we tested varying numbers for neighbors to be considered regarding the ReliefF approach. Finally, $k$ was consistently set to 10 , since it was found that results are hardly sensitive for this parameter in this study. Based on Relief-F, we compiled four feature sets, containing the 10, 20, and 50 best-ranked features, and a set with all features that have a positive degree of relevance $(\mathrm{w}>0)$. For the CFS approach, we deployed a stopping criterion of five consecutive fully expanded, nonimproving subsets. The subset with the highest merit according to the evaluation heuristic during the search was selected. Together with the thematically grouped features (sets include features from different remote sensing data sources and segmentation scales) computed features were subdivided in 11 different feature sets. For all support vector methods, we use RBF kernels (support vector methods, in this paper, were carried out with the LibSVM package by the authors of [73]).

Regarding the SVR approach, the number of samples has been varied to test sensitivity with respect to accuracy. We learn models with randomly drawn $25 \%, 50 \%, 75 \%$, and $100 \%$ 
(a)

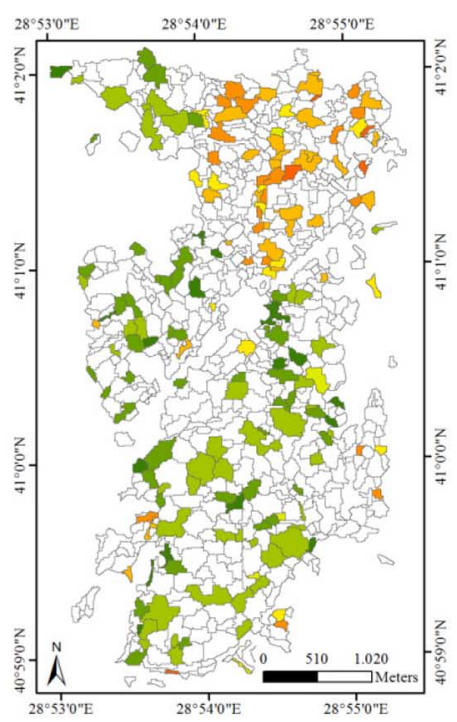

(b)

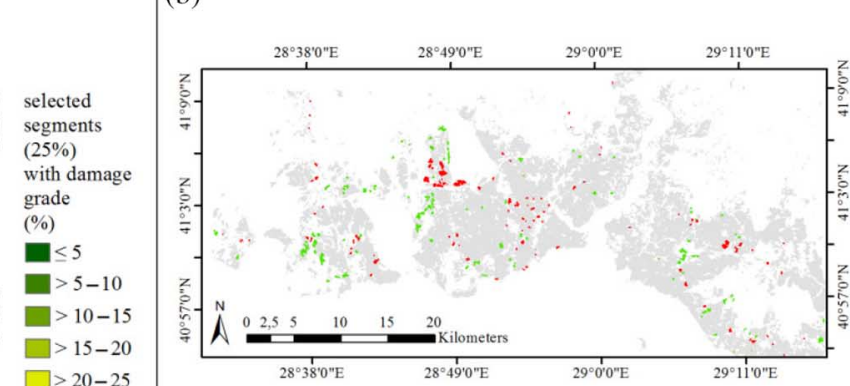

(c)
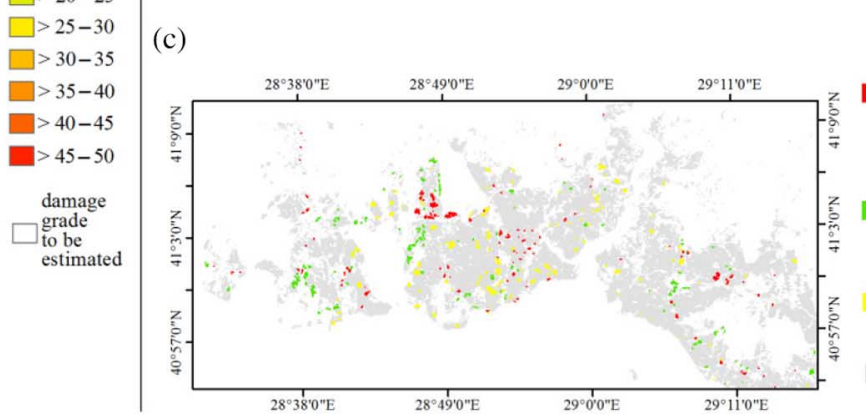

classes of interes urban structures determined by

large industrial/ (highly vulnerable) urban structures determined by tall detached residential buildings (slightly vulnerable) urban structures to be assigne to a class

urban structures determined by large industrial commercial buildings (highly vulnerable) urban structures determined by residential building (slightly vulnerable)

residual

urban structures

urban structures to be assigned to a class

Fig. 6. Concept and data for experiments. (a) Selected urban structures (25\%) with damage grades for Zeytinburnu; damage grade for residual urban structures is to be estimated with SVR. (b) Available labeled samples of classes of interest deployed for learning ensembles of $\nu$-OC-SVM. (c) Setting for $C$-SVM: additional labeled samples for classes of residual urban structures are available. This allows rendering the classification problem fully supervised.

of the labeled samples available and estimate generalization capabilities based on fivefold cross-validation. To avoid skewed results, five different training sets sharing the same number of labeled instances were created. To avoid a biased quantification of the effect of training set size on prediction accuracy, it was made sure that samples contained in one set are also contained in the affiliated set with a larger number of samples (i.e., the samples randomly drawn in one of the five sets with $25 \%$ of available labeled samples are also contained in the corresponding set with $50 \%$ of available labeled samples and so on). Generalization capability is evaluated in terms of mean absolute percentage error (MAPE) and reported as average of three independent trials (some Pearson's correlation coefficients $R$ are also reported in Section V to account for the models' goodness of fit). Model parameters were optimized with respect to MAPE in the ranges $\sigma=\left\{10^{-1}, \ldots, 10\right\}, C=\{1, \ldots, 100\}$, and $\varepsilon=\left\{10^{-6}, 10^{-3}\right\}$.

Application of the $\nu$-OC-SVM approach with a RBF kernel requires adjusting the parameter $\nu$, and the kernel-width parameter $\gamma$. Generally, it is difficult to tune free parameters if only target labeled samples are available in the training data. In such situations, solely the true positive rate (sensitivity) can be calculated, whereas the error counterpart (specificity) cannot (i.e., a suitable model cannot be distinguished from a fatally underfitted model, since also the latter would yield a high accuracy). To overcome this limitation, the free parameter selection was determined by evaluating $\arg \max _{\theta}\left\{\frac{\mathrm{OA}[\%]}{\# \mathrm{SV}}\right\}$, where $\theta$ is the set of free parameters (i.e., $\nu$ and $\gamma$ ), $\mathrm{OA}$ is the overall accuracy, and \#SV the number of support vectors. This heuristic enforces high OA while simultaneously limiting model complexity keeping a low number of $S V$ [81], [82]. For both $\nu$ and $\gamma$, we performed a grid search varying $\nu$ in the range $\{0.01, \ldots, 0.1\}$ in 0.01 steps and $\gamma$ in the range $\left\{10^{-2}, \ldots, 10^{1}\right\}$ in power of $\sqrt{10}$ steps, respectively. OA for each model was estimated by a fivefold cross-validation strategy. The parameter combination yielding the highest value of the evaluation heuristic was chosen. In case, a segment is voted as belonging to the class of interest by multiple $\nu$-OC-SVM models, reasonably, we assign the segment to the residual class, since results can be considered nondistinctive, and we generally observe considerable errors of commission in preliminary model runs. In the experiments, we used $50 \%$ of available samples for training the models and 50\% for validation. Results are reported based on five independent trials. Evaluation is based on estimated $\kappa$ statistic, which allows considering both omission and commission errors and is thus not biased by class distribution [83].

For the $C$-SVM, we adopt a one-against-one scheme [84], since we deal with more than two classes to be discriminated. Learning the most appropriate $C$-SVM in conjunction with a RBF kernel requires the definition of the cost-parameter $C$ and the kernel-width parameter $\gamma$. Tuning of $C$ and $\gamma$ was addressed by a grid search strategy based on fivefold cross-validation. Generalization accuracy is evaluated in terms of estimated $\kappa$ on the average of three independent trials. In conformity with the recommendations of the authors of [85], a grid-search with values of $C=2^{-4}, 2^{-3}, \ldots, 2^{12}$ and $\gamma=2^{-5}, 2^{-4}, \ldots, 2^{3}$ was performed. Analogous to the SVR approach, we reveal generalization capabilities as a function of feature sets with different shares of available labeled samples.

\section{EXPERIMENTAL RESULTS AND DISCUSSION}

\section{A. Estimation of Damage Grades for the District Zeytinburnu} With SVR

For Zeytinburnu, MAPEs with respect to estimated damage grades are reported in Fig. 8(a)-(c) as functions of training set size. The different figures and functions correspond to 

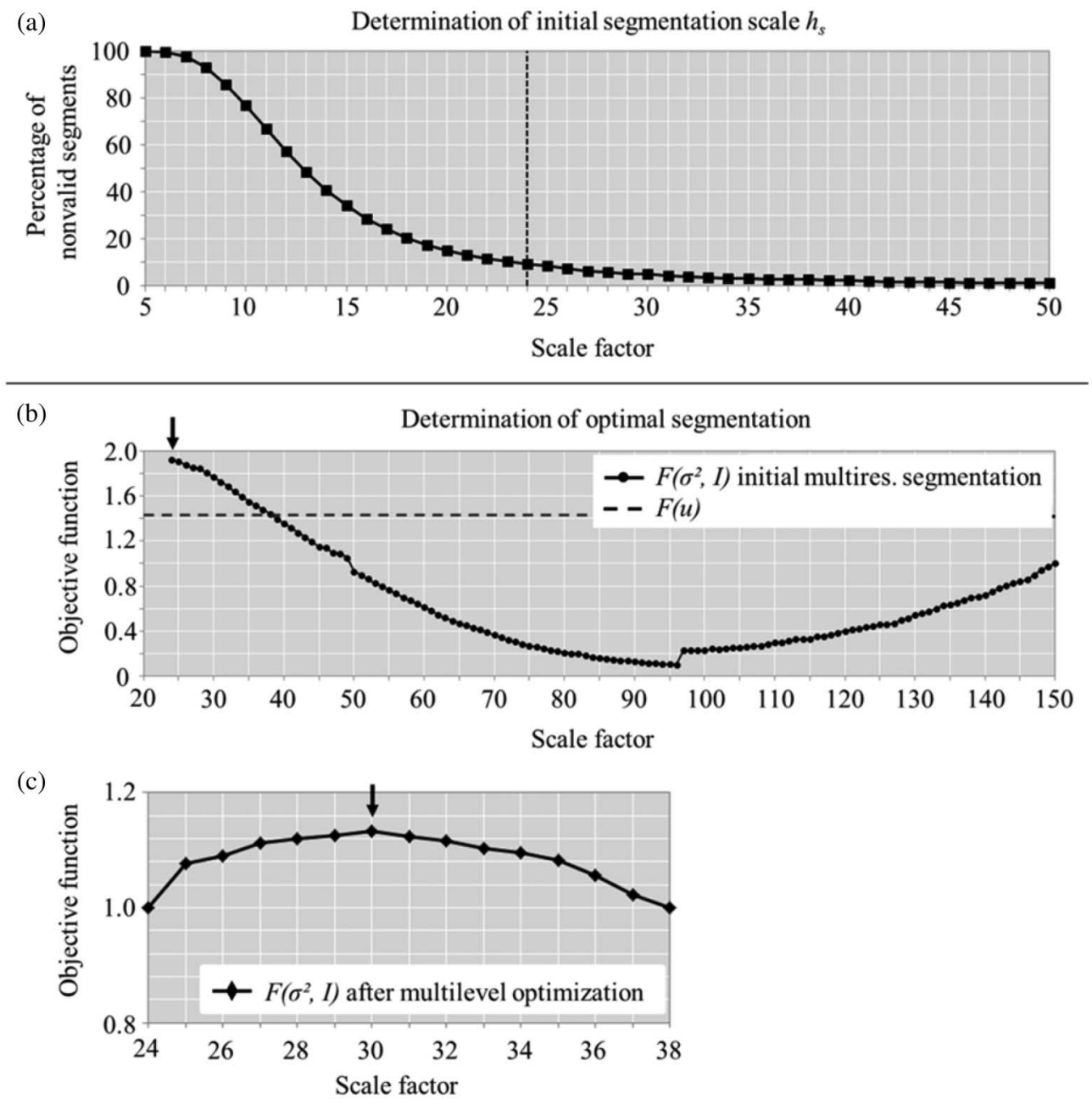

Fig. 7. Outcomes of multilevel image segmentation optimization procedure.

(a)

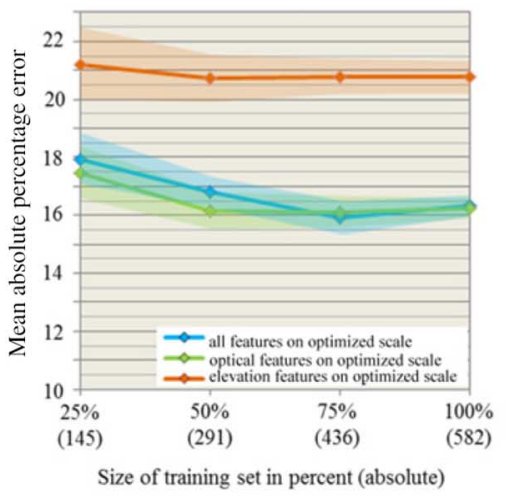

(b)

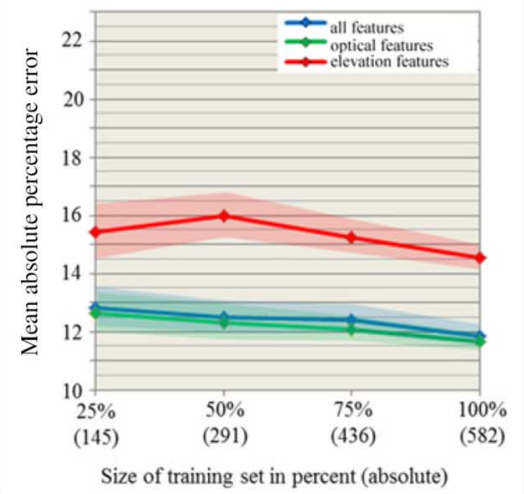

(c)

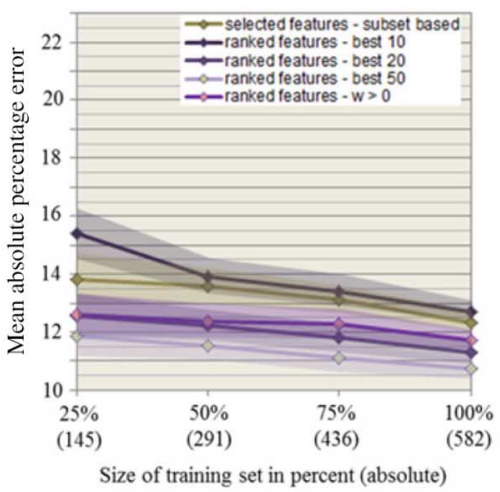

Fig. 8. MAPEs (reported as mean and standard deviation of different model runs) as a function of training set size. (a) MAPE obtained with features from the optimized segmentation level only. (b) MAPE obtained with features from all three segmentation levels. (c) MAPE obtained with features selected by filter methods.

MAPE obtained with different feature sets. Generally, it can be noted that graphs reveal distinctive differences with respect to accuracy. When using solely features calculated from the elevation data (i.e., nDSM), MAPE are considerably higher than corresponding MAPE obtained with features computed from the optical data [Fig. 8(a)]. In particular, MAPE decrease from $20.76 \%( \pm 0.94 ; R=0.163)$, achieved with elevation features, to $16.23 \%$ ( $\pm 0.36 ; R=0.544)$, achieved with the optical features, when all samples were deployed within the crossvalidation procedure. Analogously, when examining accuracies obtained with features computed not only from the optimized segmentation level but also from supersegments [Fig. 8(b)], we 
(a)

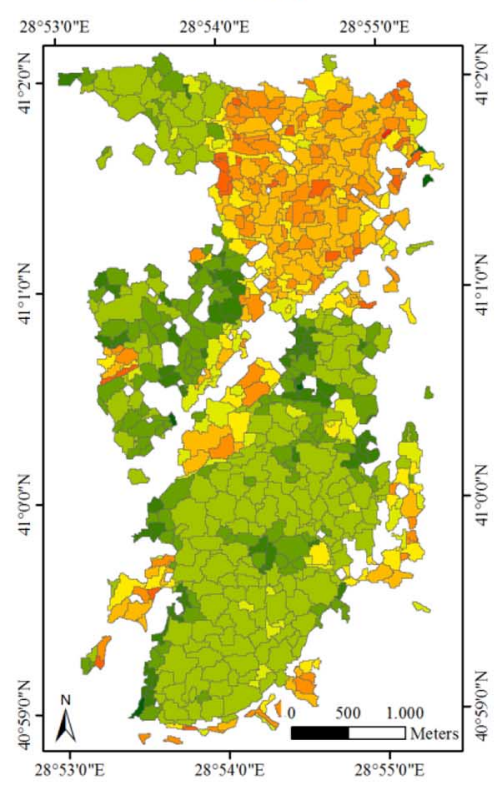

\section{Zeytinburnu}

damage grade

(\%)

$\leq 5$

$\square>5-10$

$>10-15$

$>15-20$

$>20-25$

$\square>25-30$

$\square>30-35$

$>35-40$

$>40-45$

$>45-50$

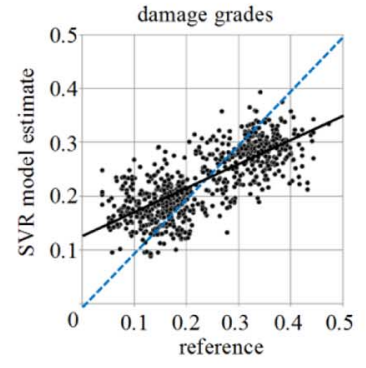

Model was learned with $50 \%$ of available labeled samples

and $50 \%$ were held out for validation. A MAPE of $13.00 \%$ and $R$ of 0.754 wa

obtained with 50

features ranked

highest with Relief-F.

Istanbul

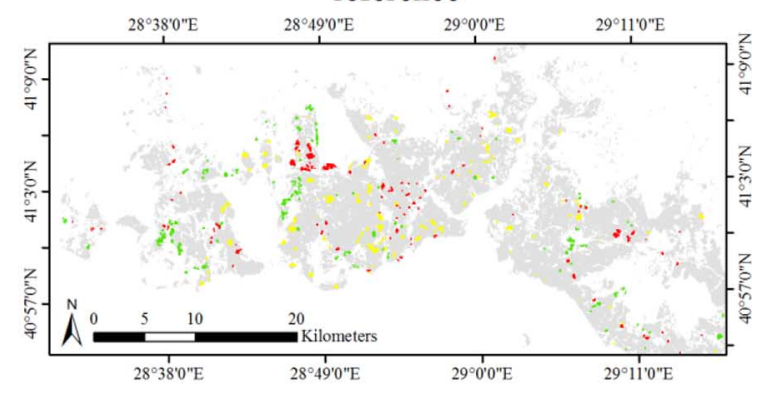

ensemble of $v$-OC-SVM

(b)

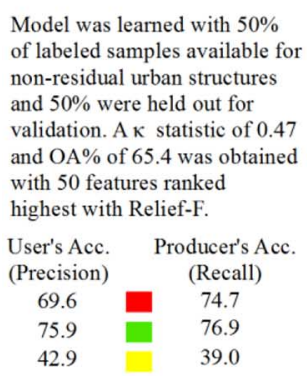

(c)

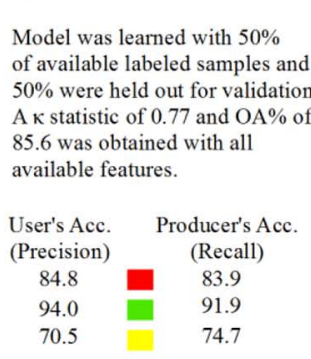

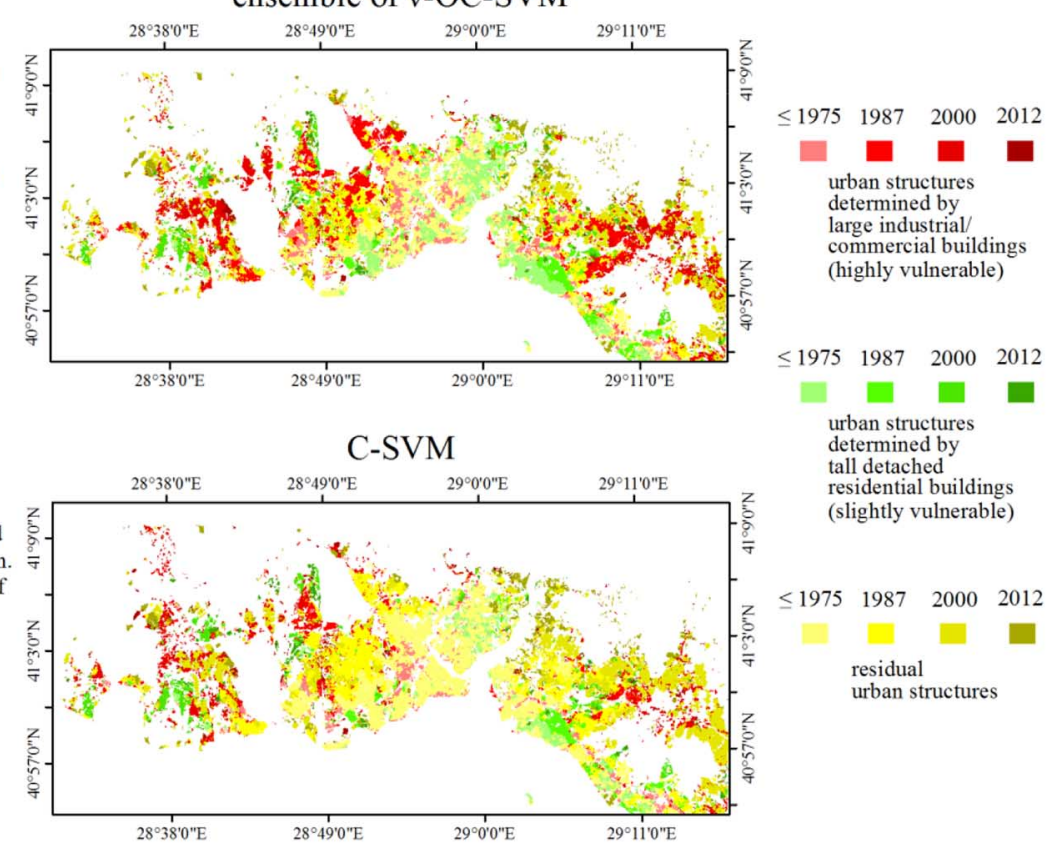

Fig. 9. Application of learned models. (a) Estimated damage grade with SVR for Zeytinburnu. (b) Classified urban structures of Istanbul according to an ensemble of $\nu$-OC-SVM. (c) Classified urban structures of Istanbul according to a fully supervised $C$-SVM. 
also observe a considerable decrease in MAPE from $14.54 \%$ $( \pm 0.41 ; R=0.596)$ to $11.64 \%( \pm 0.30 ; R=0.753)$. Besides, an explicit gain of accuracy can be observed when incorporating supersegment information compared to a single segmentation (e.g., decrease of MAPE with both optical and elevation features from $16.34 \%$ to $11.85 \%$; simultaneously, goodness of model fit raises from $R=0.521$ to $R=0.741$ ). Fig. 8 (a) and (b) also reveals that a joint use of optical and elevation information does not increase accuracy remarkably compared to accuracies obtained with optical features alone (difference in MAPE is $0.36 \%$ and $0.30 \%$, respectively). Finally, Fig. 8(c) shows that the application of filter methods allow to identify beneficial feature sets for the estimation of damage grades. Thereby, lowest MAPE of all feature sets (i.e., 10.74\%; $R=$ 0.778 ) could be obtained with the 50 highest ranked features from the Relief-F algorithm.

These numerical results suggest that features from optical data allow estimating damage grades with viable accuracies, whereas elevation features, as derived from the TDM data, do not allow estimating damage grades in a viable way. Here, also a joint exploitation of both data sets does not feature a clear improvement in accuracy. In contrast, the idea to exploit supersegment information proved to enhance accuracy of estimates considerably. This is in line with previous experimental analyses that confirm an increase of prediction accuracy, when modeling spatial context information in feature space with supersegment information (e.g., [62] and [63]). However, this observation simultaneously suggests that homogeneous urban structures could not consistently be discriminated properly based on the optimized segmentation, and that oversegmentation has occurred. However, in contrast to under-segmentation, oversegmentation leaves the possibility to gain accurate mapping results after classification (i.e., a possibly over-segmented urban structure, which may be represented by two segments instead of one, can be mapped properly if both segments are assigned to the same class). In this sense, the incorporation of supersegment information allowed to gain viable accuracies in the experiments. In addition, deployed feature selection algorithms proved useful to alleviate problems associated with high-dimensional feature vectors in conjunction with a comparatively small number of labeled samples, since best accuracies were achieved with subsets of the features. Thereby, best models are characterized by MAPE less than $11 \%$ and fairly strong goodness of fit $(\mathrm{R}>0.75)$.

As a further means, an application of a model to estimate damage grades for Zeytinburnu is shown in Fig. 9(a). It can be noted that a further differentiation according to categories of construction periods is not provided, since the settlement area of Zeytinburnu was consistently covered before 1975 . The model was learned with $50 \%$ of available labeled samples and $50 \%$ were held out for validation. A MAPE of $13.00 \%$ and $R$ of 0.754 were obtained based on 50 features ranked highest with Relief-F. Generally, a good spatial agreement of reference and model estimates can be observed. Areas characterized by high expected damage grades in the northern part of Zeytinburnu feature also highest model estimates. Instead, a vast majority of the central and southern parts correspond to moderate or low damage grades in both maps. Simultaneously, as can be seen

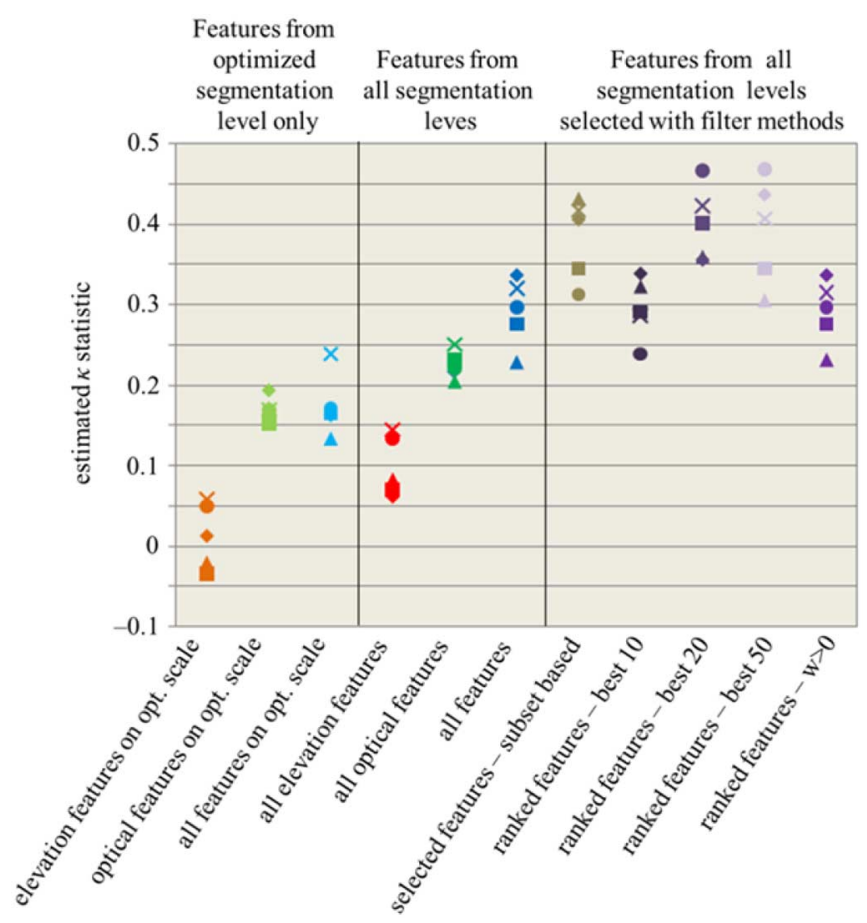

Fig. 10. $\kappa$ statistic obtained with an ensemble of $\nu$-OC-SVM for different feature sets. Samples were split in a stratified manner and models were learned with $50 \%$ of available samples and $50 \%$ were used for validation; five different model runs with a differing composition of samples were carried out (marked with different symbols, i.e., $\mathrm{x}, \circ, \Delta, \diamond$, and $\square$ ).

from the scatter plot, the model overestimates damage grades, for instances, with low damage grades, and underestimates high damage grades.

\section{B. Assignment to Classes of Interest With an Ensemble of $\nu$-OC-SVM}

We aimed to identify two distinctive urban structures (i.e., urban structures determined by large industrial/commercial buildings that can be considered as highly vulnerable, and urban structures determined by tall detached residential buildings that can be considered as slightly vulnerable) within the settlement area of Istanbul, disregarding all other potentially present classes in the area. Only labeled samples were available for the classes of interest in this experiment and not for all classes present in the data, which rendered the prediction problem very challenging.

Prediction accuracies, as evaluated with estimated $\kappa$ statistics for different feature sets and model runs, are shown in Fig. 10. Analogous to results of SVR, we observe considerable differences in accuracy with respect to deployed feature sets. It can be noticed that $\kappa$ statistics achieved with features from the optimized segmentation level indicate agreements just slightly better than chance (i.e., $\kappa$ statistics vary from -0.03 to 0.24). Again, elevation features used alone perform worst. Interestingly, when incorporating knowledge from supersegments, a consistent increase of interrater agreement can be observed for all three feature groups. Corresponding $\kappa$ statistics show a mean increase in terms of absolute values by $0.09,0.05$, and 0.12 , respectively. Thereby, the unreduced feature vector 
(a)

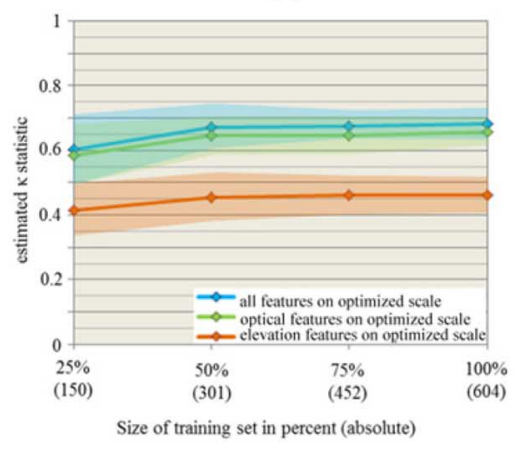

(b)

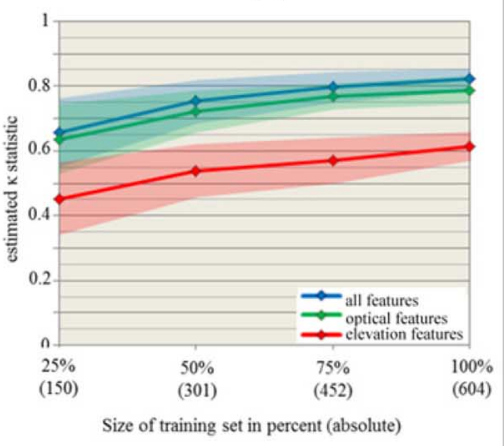

(c)

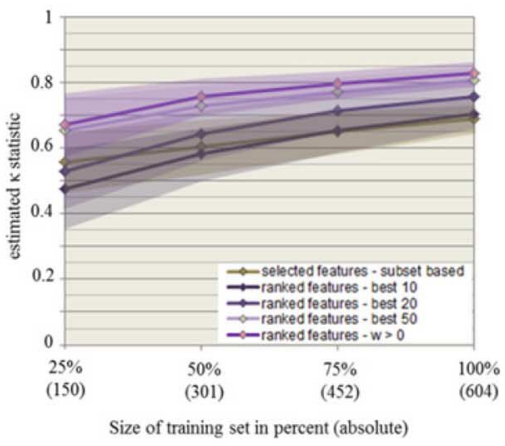

Fig. 11. $\kappa$ statistics (reported as mean and standard deviation of different model runs) as a function of training set size. (a) $\kappa$ obtained with features from the optimized segmentation level only. (b) $\kappa$ obtained with features from all three segmentation levels. (c) $\kappa$ obtained with features selected by filter methods.

allows to exhibit fair agreements with $\kappa$ statistics between 0.23 and 0.34. Nevertheless, applied strategies to reduce dimensionality of the feature vector proved useful, since features sets generated with filter methods generally provide highest $\kappa$ statistics. Best models feature a moderate agreement, with $\kappa$ statistics larger than 0.45 .

Interestingly, in accordance with the findings regarding the SVR approach, also here, we observe that elevation features used alone yield least favorable results. However, in contrast to SVR, a joint exploitation with optical features yields a substantial improvement in accuracy when simultaneously considering supersegment information (mean $\kappa$ statistics increase from 0.17 to 0.29 ). Results are comparable to those obtained with models learned from filter-based features sets. In this sense, feature selection algorithms proved useful again, since best results were obtained with 20 and 50 features ranked highest with Relief-F. However, $\kappa$ statistics cannot exhibit a moderate agreement (i.e., best models feature $\kappa$ statistics between 0.4 and 0.47 ), which questions viability in real-life situations. Although benchmark accuracies that need to be met in real-life situations are largely absent within this rather emerging application context, results correspond to the challenging nature of the prediction problem.

An ensemble of models was applied to assign urban structures of Istanbul to the classes of interest [Fig. 9(b)]. Additionally, they are further differentiated according to the categories of established construction periods. The ensemble of models was learned with $50 \%$ of labeled samples available for nonresidual urban structures and 50\% were held out for validation. A $\kappa$ statistic of 0.47 and $\mathrm{OA} \%$ of 65.4 were obtained based on 50 features ranked highest with Relief-F. From the affiliated user's and producer's accuracies, it can be seen that urban structures determined by tall detached residential buildings could be identified most accurately, closely followed by urban structures determined by large industrial/commercial buildings. The residual class shows lowest accuracies. This result can be reasonably related to the peculiarities of the different classes, whereby, e.g., structures of tall detached residential buildings are constituted by most distinctive characteristics. Instead, the residual class features a large variety of different structures. Nevertheless, large parts of the settlement area were assigned to either nonresidual class. The validity of this vast assignment can be questioned, in particular, in conjunction with results from the subsequent experiment (discussion follows at the end of the subsequent section). In turn, this suggests a large error of commission, evolving from broad, nonsufficient decision functions of the models describing the distributions of the two classes of interest.

\section{Assignment to Classes of Interest With C-SVM}

To complement results obtained with an ensemble of $\nu$-OC$\mathrm{SVM}$, we also rendered the prediction problem fully supervised and approached it with $C$-SVM. Results are reported in terms of estimated $\kappa$ statistics in Fig. 11(a)-(c). As for the two previous experiments, also here, we observe considerable differences with respect to accuracy in dependence of deployed feature sets. When using solely elevation features computed from the optimized segmentation level, $\kappa$ statistics feature a fair to moderate agreement (i.e., mean $\kappa$ statistics vary between $0.42 \pm 0.09$ and $0.46 \pm 0.05)$. In contrast, optical features allow achieving substantial $\kappa$ statistics larger than 0.6 most of the time [Fig. 11(a)]. Again, usability of supersegment information is expressed in a further increase of $\kappa$ statistics, which definitely met a substantial agreement (i.e., $\kappa>0.6$ ) when using optical features or jointly exploit optical and elevation features [Fig. 11(b)]. Thereby, a joint use of optical and elevation features yields an increase of $\kappa$ between 0.022 and 0.044 compared to the use of optical features alone. In this sense, best models show excellent $\kappa$ statistics larger than 0.8. Regarding feature sets composed with filter methods, we observe that only a sufficient number of features ensure viable $\kappa$ statistics with a substantial or excellent agreement. Thereby, features that show a positive degree of relevance as evaluated with Relief-F $(\mathrm{w}>0$; $n=152$ ) consistently performed best [Fig. 11(c)].

Results obtained with $C$-SVM show distinctive increase of accuracies when compared to results obtained with ensembles of $\nu$-OC-SVM. Ensembles of $\nu$-OC-SVM could not exceed moderate agreements, with $\kappa$ statistics slightly above 0.45 . Instead, $C$-SVM allowed to obtain $\kappa$ statistics showing substantial and even excellent agreement $(\kappa>0.6$ up to $\kappa>0.8)$. Generally, this is reasonable and meets expectations, since $C$ SVM can rely on more prior knowledge. In fact, it is much more challenging to estimate the support of multiple multi- or 
high-dimensional distributions, than to discriminate known target classes from each other. Nevertheless, the use of labeled samples from classes others than the classes of interest proved very useful to achieve accuracies that may be needed in real-life cases. Hence, results confirm viability of proposed procedures to support an assessment of urban structures with respect to their seismic vulnerability.

The application of a $C$-SVM model to assign urban structures of Istanbul to the classes of interest is shown in Fig. 9(c). It was learned with $50 \%$ of available labeled samples and $50 \%$ were held out for validation. A $\kappa$ statistic of 0.77 and OA\% of 85.6 were obtained with all available features. User's and producer's accuracies reveal that urban structures determined by tall detached residential buildings could be identified very accurately (accuracies $>90.0$ ), and also urban structures determined by large industrial/commercial buildings feature very good agreements (accuracies $\sim 84.0$ ). A moderate decrease in accuracy can be observed for the class of residual urban structures (user's acc. of 70.5 and producer's acc. of 74.7). These different class-related levels of accuracy are unambiguously in line with the previous experiment. However, in distinction to the results obtained with an ensemble of $\nu$-OC-SVM, the majority of the settlement area of Istanbul is assigned to residual urban structures and urban structures of particular interest emerge now in a spatially very explicit way. As just one example, consider urban structures determined by large industrial and commercial buildings in the southern part of the Asian side in Fig. 9(c). Those clearly emerge in a linear manner, in reality, along an arterial road.

Finally, it should be noted that we addressed in the experiments a comparatively coarse level of abstraction regarding the vulnerability classes to be estimated, e.g., we aimed to identify urban structures determined by large industrial/commercial buildings, which can be considered as highly vulnerable in our application. However, especially industrial structures can feature a considerable variety in the main structural parameters that are relevant for vulnerability assessment, such as the lateral load resisting system, or construction material. Nevertheless, accurate identification of structures determined by large industrial/ commercial buildings can be helpful to identify priority areas and serve as a basis for a guided and more detailed reassessment.

\section{Summary, CONCLUSION, AND Future Perspectives}

In this paper, we suggested procedures that learn prior seismic vulnerability information to estimate seismic vulnerability levels of urban structures based on remote sensing data. In contrast to numerous studies that operate on building level, we, therefore, addressed a coarser level of the urban morphology. This allowed relying on remote sensing data with a lower spatial resolution, but larger spatial coverage. In this sense, we exploited data from the RapidEye constellation, TDM, and Landsat archive.

We provided numerical results obtained for the city of Istanbul confirm viability of procedures. When estimating damage grades for Istanbul's district Zeytinburnu with SVR, best models were characterized by MAPEs less than $11 \%$ and fairly strong goodness of fit $(\mathrm{R}>0.75)$. When aiming to identify types of urban structures (i.e., urban structures determined by large industrial/commercial buildings that can be considered as highly vulnerable in this application, and urban structures determined by tall detached residential buildings that can be considered as slightly vulnerable), results obtained with $C$-SVM showed distinctive increase of accuracies when compared to results obtained with ensembles of $\nu$-OC-SVM. This is reasonable since $C$-SVM could rely on more prior knowledge. Ensembles of $\nu$-OC-SVM were not able to exceed moderate agreements, with $\kappa$ statistics slightly above 0.45 . Instead, $C$-SVM allowed to obtain $\kappa$ statistics showing substantial and even excellent agreements $(\kappa>0.6$ up to $\kappa>0.8)$.

Besides, during experiments, we observed that elevation information, as provided by the nDSM, used alone does not allow for learning viable models. However, when jointly exploited with optical data, learned models exhibit viable accuracies. It is worth noting that the idea to model spatial context relations in feature space with supersegment information has also shown very favorable results and in most cases enabled to retrieve viable accuracies at all. In this regard, feature selection algorithms proved generally useful to alleviate problems associated with high-dimensional feature vectors in conjunction with a comparatively small number of labeled samples. All in all, analyses provide promising empirical evidence, which confirms the potential of remote sensing to support the seismic vulnerability assessment of urban structures.

However, from a methodological perspective, future work may exploit semisupervised approaches (e.g., [86]), which encode some knowledge from the unlabeled data also. They may yield favorable accuracies especially in situations where only very few labeled samples are available. Ascertainment of labeled samples can generally be considered to be very costly within this application context. Therefore, active learning strategies [87], [88] may be followed to solve estimation of vulnerability levels efficiently, based on remote sensing data in a supervised fashion. To this purpose, predefined heuristics can be deployed to rank unlabeled instances in the domain under analysis that are considered the most valuable for improvement of estimation accuracy of a preliminary model learned with available prior seismic vulnerability knowledge. Once instances are selected, they are labeled and the learning process is iterated. Recent approaches also include the spatial domain for this task [89], [90], and consider labeling costs emerging from ground surveys [91]. Such approaches may guide civil engineers through the building inventory to collect most valuable additional in situ information efficiently and ensure simultaneously that there is no sample selection bias [92]. Moreover, the incorporation of classification uncertainty measures can be deployed to iteratively assess and redefine the sample sites [93]. Before, the concept of focus maps [94] allows prioritizing areas for data collection with respect to multiple criteria (e.g., hazard probability, (quality of) available data, etc.) in an integrative way.

The idea of a targeted estimate, i.e., identifying the classes of interest by having only labeled samples of the classes of interest available and not for all classes present in the data, should be further followed. In this paper, we deployed ensembles of $\nu$-OC-SVM for this task. Thereby, identification of 

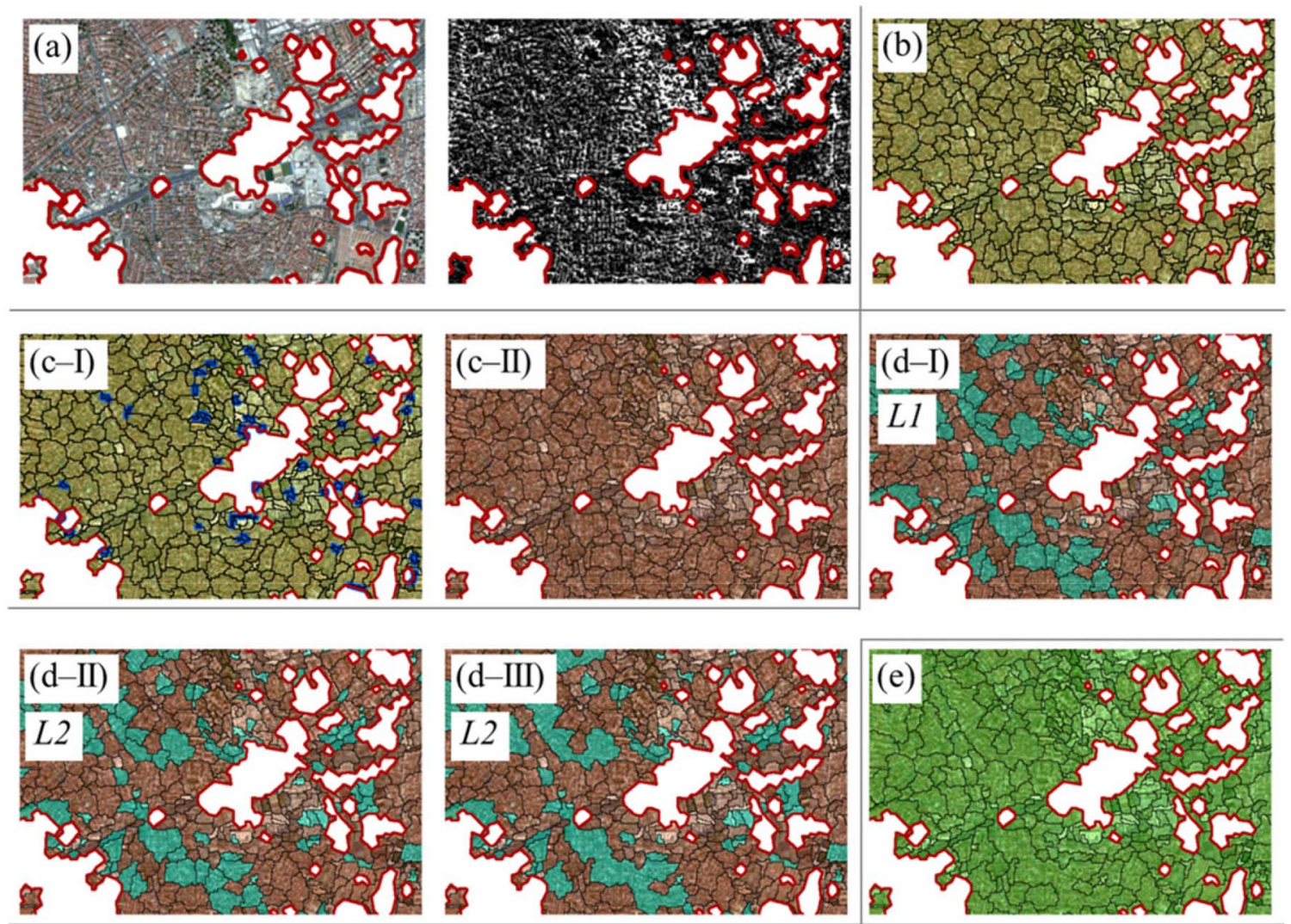

— Urban footprint (UF)

Initial multiresolution segmentation (ioMRS)

Non-valid segment (nvS)
Initial multiresolution

segmentation merged (ioMRS-m)

Real subsegment (rSS)

Final segmentation (fS)

Fig. 12. Illustration of basic processing steps of the image segmentation procedure. (a) Incorporation of the UF as basic segmentation level visualized for both multispectral and nDSM data. (b) Initial optimal multiresolution segmentation (ioMRS) is determined with a plateau objective function. (c-I) Segments are identified that do not correspond to the Shannon sampling theorem and are considered as nvSs. (c-II) nvSs are merged with most similar adjacent segments (ioMRS-m). (d-I) Distinctive subsegments (rSS) are determined and transferred to the (d-II) supersegment level. (d-III) They are merged if a similarity constraint is fulfilled. (e) Final segmentation (fS) is identified by reassessment with the objective function after multilevel segmentation procedure.

most suitable hyperparameters is generally very difficult and we used a heuristic, which evaluates overall accuracy and model complexity, expressed by number of support vectors. However, more sophisticated approaches may identify more suitable combinations of free parameters (e.g., approaches that aim to characterize and assess the shape of the decision function of learned models). In addition, unsupervised preclassification of the feature space has shown promising results recently [95]. Besides, other machine learning one-class approaches (e.g., ensembles of one class random forests [96]) can be considered as an interesting opportunity. Regarding alternative techniques for function estimation, Gaussian process regression [97] has shown distinctive accuracies recently, when compared to other machine learning regression techniques (e.g., [98]). At the same time, it is generally crucial to rely on techniques that ensure a high transferability of trained learning machines. In the context of multispectral image classification, the authors of [99] showed recently that, e.g., SVM can be considered as a viable approach.

From a conceptual point of view, an intensive exchange with the earthquake engineering community appears exigent.
Definition of typologies that need to be estimated in a standardized way and commitment of benchmark accuracies that need to be met would allow a rigorous evaluation of approaches. This may contribute to a further determination of the role of remote sensing within this emerging application field.

\section{APPENDIX A}

This section is used to visualize some basic processing steps of the image segmentation procedure (Fig. 12). Based on joint usage of the UF data set, multispectral, and height information, an initial optimal segmentation layer is obtained. Validity measures are applied to modeled segments and segmentation results are adjusted according to predefined criteria. Subsequently, a multiscale optimization is conducted and an optimal multiscale segmentation level is finally identified from the outcomes.

\section{APPENDIX B}

Fig. 13 shows the general principle of nonlinear SVM. Data are mapped with a kernel function from the input space $\chi$ to 


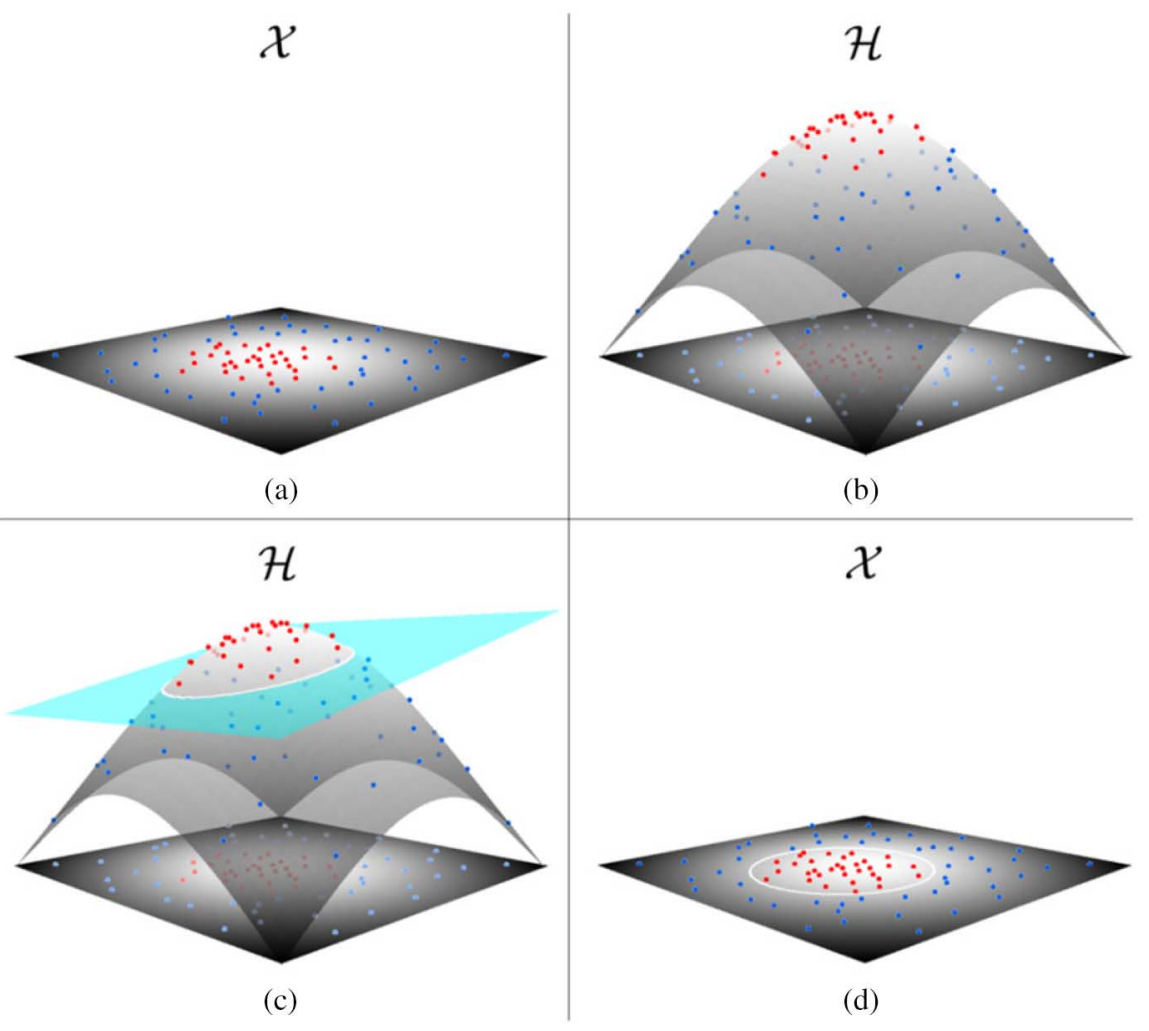

Fig. 13. Idealized procedure for generation of a nonlinear decision function by SVM. (a) Dataset with two classes (red and blue dots) that are nonlinearly separable in $\chi$ are mapped through a nonlinear transformation $\phi(\cdot)$ into a space of (b) higher dimensionality $\mathcal{H}$. (c) Linear separation becomes possible in that space and a hyperplane (cyan) with maximum margin is fitted, which corresponds to a (d) nonlinear decision function in $\chi$.

a space of higher dimensionality $\mathcal{H}$, where a linear separation becomes possible. The solution corresponds to a nonlinear separation in the original input space.

In Fig. 14, three different formulations of SVM are visualized. Those correspond to multiclass classification problems [Fig. 14(a)], one-class classification problems [Fig. 14(b)] and regression problems [Fig. 14(c)].

\section{Multiclass Classification With $C$-SVM}

The $C$-SVM method was introduced by the authors of [100] to cope with class overlap or the existence of noise in the training data in multiclass problems. This technique represents a modification of the maximum margin approach using relaxed separation constraints that allow for the possibility of instances on the incorrect side of the respective margin boundary. Let us consider a data set with labeled instances $\left\{\mathbf{x}_{i}, y_{i}\right\}_{i=1}^{n}$, with $\mathbf{x}_{i} \in \mathbb{R}^{d}$ and $y_{i} \in\{-1,+1\}$. The data is mapped through a nonlinear transformation $\phi(\cdot)$ to a space with a higher dimension. An appropriate determination of $\phi(\cdot)$ ensures that the transformed samples are more likely to be linearly separable in the higher dimensional space [77]. Then, the minimization objective of $C$-SVM is formulated as

$$
\min _{\mathbf{w}, \xi_{i}, b}\left\{\frac{1}{2}\|\mathbf{w}\|^{2}+C \sum_{i=1}^{n} \xi_{i}\right\}
$$

subject to

$$
\begin{aligned}
y_{i}\left(\phi\left(\mathbf{x}_{i}\right), \mathbf{w}+b\right) \geq 1-\xi_{i} \quad \forall i=1, \ldots, n \\
\xi_{i} \geq 0 \quad \forall i=1, \ldots, n
\end{aligned}
$$

where $\mathrm{w}$ represents the normal perpendicular to the optimal separating hyperplane and $b$ is the nearest distance to the origin $(\mathcal{O})$ of the coordinate system. These parameters constitute a linear classifier in $\mathcal{H}$, which separates the labeled samples of different classes with maximum margin. To enhance generalization capabilities and reduce over-fitting, positive slack variables $\xi_{i}$ are introduced to account for labeled samples lying on the incorrect side of the respective margin boundary [Fig. 14(a)]. The constant $C$ determines the trade-off between maximizing the margin and the number of incorrectly classified samples (training errors). An optimal parameterization of $C$ can be determined empirically. It can be noted that (12) is constituted by two distinctive terms that are clearly interpretable: the objective is to minimize simultaneously both the norm of the model weights $\left\|\mathbf{w}^{2}\right\|$, which is equivalent to the maximization of the margin, and the committed errors $\sum_{i=1}^{n} \xi_{i}$ [80].

The minimization objective of (12) is reformulated from its primal form to its dual form by introducing Lagrange multipliers, so that it can be solved with quadratic programming techniques efficiently [74]. Finally, a decision function is given 


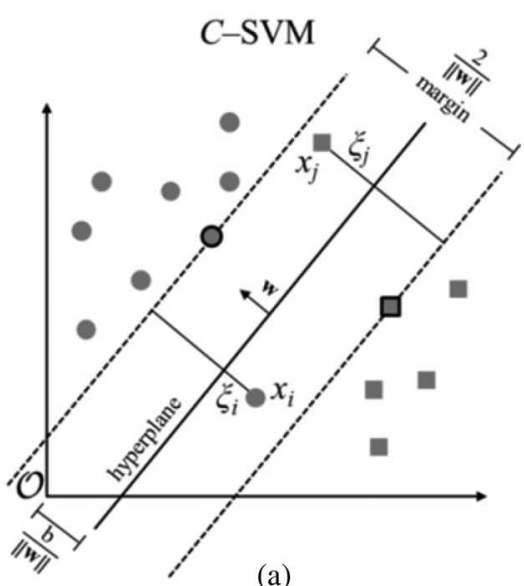

(a)

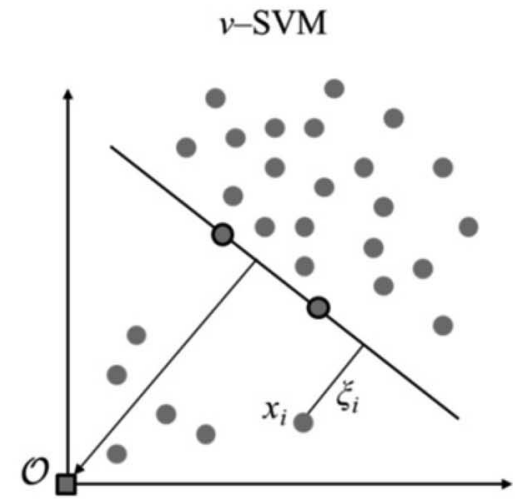

(b)

\section{SVR with Vapnik's \\ $\varepsilon$-insensitive cost function}

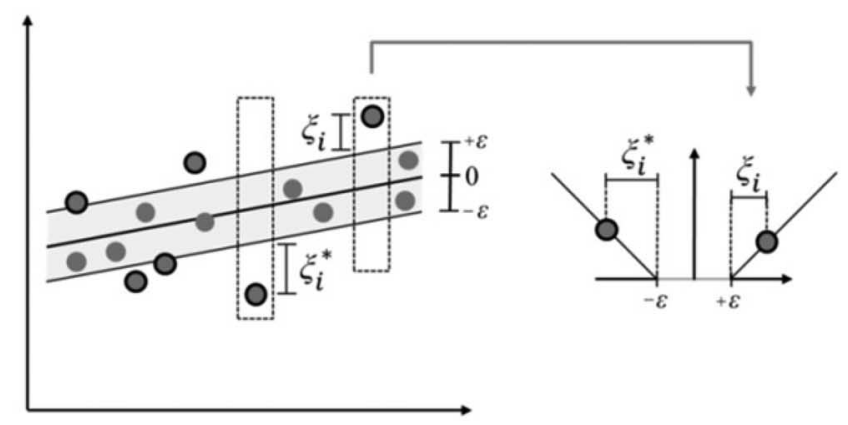

(c)

Fig. 14. Different formulations of SVM. (a) $C$-SVM provide a linear decision function with relaxed separation constraints, which allow for labeled samples lying on the incorrect side of the hyperplane (modified adaption from the authors of [76]). (b) $\nu$-OC-SVM treat the origin of the feature space $(\mathcal{O})$ as the only available sample of the nontarget class first, and fit a hyperplane with maximum margin from the origin (modified adaption from the authors of [81]). (c) In SVR, all samples outside a fixed tube with size $\varepsilon$ (i.e., support vectors) are penalized by applying a cost function. Here, Vapnik's $\varepsilon$ cost function is deployed, which accounts for a linear penalization (modified adaption from the authors of [74], [80]).

that allows assigning a class label to an instance of unknown class membership $\mathbf{x}_{*}$

$$
f\left(\mathbf{x}_{*}\right)=\operatorname{sgn}\left(\sum_{i=1}^{n} y_{i} \alpha_{i} K\left(\mathbf{x}_{i}, \mathbf{x}_{*}\right)+b\right)
$$

with $\alpha_{i}$ being the Lagrange multipliers and $K$ being a kernel function. The Lagrange multipliers are determined by optimization and feature nonzero values for instances lying on the margin-the support vectors [77], [78], [100]. The kernel function $K$ is expressed as the dot product of mapped instances $K\left(\mathbf{x}_{i}, \mathbf{x}_{j}\right)=\phi\left(\mathbf{x}_{i}\right), \phi\left(\mathbf{x}_{j}\right)$. Hence, the outcome of the decision function (15) only relies on the dot product of the vectors in the input space $\chi$. This allows to avoid an explicit projection to a space of higher dimensionality, since this formulation yields the same result (also known as the kernel trick). This property of the SVM algorithm enables a very efficient computation of decision functions for data with a very high dimensionality. A number of different kernels with varying characteristics exist. However, in environmental applications, it is common to use Gaussian RBF kernels, which take the form $K\left(\mathbf{x}_{i}, \mathbf{x}_{j}\right)=\exp \left(-\left\|\mathbf{x}_{i}-\mathbf{x}_{j}\right\|^{2} / 2 \sigma^{2}\right)$, due to their interpretability (they express local similarity) in accordance with favorable performance properties [71].

\section{ONE-Class Classification With $\nu$-SVM}

$\nu$-OC-SVM were introduced by the authors of [101] as support vector method for novelty detection. It can be deployed in situations where the objective is to identify only one or few classes of interest from all classes present in the data, simultaneously having only labeled samples of the classes of interest available.

Generally, the strategy of $\nu$-OC-SVM is to capture the support region (i.e., where the density is large) without the need of prior assumptions about the distribution of the data. Therefore, the target class is described by a function that maps the majority of instances to a region where the function is nonzero. To achieve this, the origin of the feature space is first treated as the only available member of the nontarget class (i.e., as an outlier). Then, a hyperplane with maximum margin separation from the origin is identified [Fig. 14(b)]. To separate the data from the origin, the minimization objective is as follows:

$$
\min _{\|\mathbf{w}\|, \xi_{i}, p}\left\{\frac{1}{2}\left\|\mathbf{w}^{2}\right\|+\frac{1}{\nu l} \sum_{i} \xi_{i}-p\right\}
$$


subject to

$$
\begin{aligned}
&\left\langle\mathbf{w}, \phi\left(\mathbf{x}_{i}\right)\right\rangle \geq p-\xi_{i} \forall i=1, \ldots, l \\
& \xi_{i} \geq 0 \quad \forall i=1, \ldots, l
\end{aligned}
$$

where $\mathbf{w}$ represents a vector, which is perpendicular to the hyperplane, and $p$ is the distance to the origin. Parameter $\nu \in$ $(0,1]$ controls the trade-off between an upper bound of fraction of margin errors (as can be seen from (16), outliers in the training data are handled by slack variables analogous to the $C$-SVM framework) and a lower bound of fraction of support vectors (i.e., model complexity) [81], [102]. Again, by utilizing Lagrange multipliers and a kernel function, the final decisions function to assign a class label to an instance of unknown class membership $\mathbf{x}_{*}$ is obtained by

$$
f\left(\mathbf{x}_{*}\right)=\operatorname{sgn}\left(\sum_{i} \alpha_{i} K\left(\mathbf{x}_{i}, \mathbf{x}_{*}\right)-p\right) .
$$

In practice, $\nu$-OC-SVM can be used in problems when more than a single specific class of interest is considered at a time by employing an ensemble of one-class classifiers. Thereby, each classifier is trained on a specific class of interest. When manifold classifiers assign a class label to an instance, a heuristic can be applied to the outputs (e.g., a winner-take-all rule based on prior or posterior probabilities) to determine the final class membership [82].

\section{Function Estimation With SVR}

SVR allows approximating a function from training data, when the statistical level of measurement of the target variable corresponds to an interval or ratio scale. In accordance to the presented SVM framework, SVR defines a linear model over samples that are mapped to a higher dimensional space via a nonlinear function. A common SVR formulation deploys Vapnik's $\varepsilon$-insensitive cost function, in which errors up to $\varepsilon$ are not penalized, and further errors are subject to a linear penalization [Fig. 14(c)]. Thereby, SVR determines weights w by minimizing a regularized functional

$$
\min _{\|\mathbf{w}\|, \xi_{i}, \xi_{i}^{*}, b}\left\{\frac{1}{2}\left\|\mathbf{w}^{2}\right\|+C \sum_{i}\left(\xi_{i}+\xi_{i}^{*}\right)\right\}
$$

subject to

$$
\begin{aligned}
y_{i}-\left(\left\langle\phi\left(\mathbf{x}_{i}\right), \mathbf{w}\right\rangle+b\right) \leq \varepsilon+\xi_{i} & \forall i=1, \ldots, n \\
\left(\left\langle\phi\left(\mathbf{x}_{i}\right), \mathbf{w}\right\rangle+b\right)-y_{i} \leq \varepsilon+\xi_{i}^{*} & \forall i=1, \ldots, n \\
\xi_{i}, \xi_{i}^{*} \geq 0 & \forall i=1, \ldots, n
\end{aligned}
$$

where $\xi_{i}$ and $\xi_{i}^{*}$ are positive slack variables, which quantify the distances of the labeled training samples that lie outside of the $\varepsilon$-insensitive tube to the boarder of the tube [Fig. 14(c)]. The regularization parameter $C$ determines the trade-off between the flatness of the function and the tolerance to observed errors. Similar to the previous formulations, the minimization objective can be solved by introducing linear restrictions (21)(23) into (20) using Lagrange multipliers $\alpha_{i}$, calculate the Karush-Kuhn-Tucker conditions, and solve the dual problem with quadratic programming techniques [74], [80], [97], [103]. Subsequently, the final estimation function is given by

$$
f\left(\mathbf{x}_{*}\right)=\sum_{i=1}^{n}\left(\alpha_{i}-\alpha_{i}^{*}\right) K\left(\mathbf{x}_{i}, \mathbf{x}_{*}\right)+b .
$$

\section{ACKNOWLEDGMENT}

The authors would like to thank A. Felbier and T. Esch (DLR) for computing and providing the urban footprint data, and the TanDEM-X science team for providing TDM intermediate DSM data for the test site. They would also like to thank the anonymous reviewers for their helpful comments and suggestions.

\section{REFERENCES}

[1] R. Bilham, "The seismic future of cities," Bul. Earthquake Eng., vol. 7, pp. 839-887, 2009.

[2] T. L. Holzer and J. C. Savage, "Global earthquake fatalities and population," Earthquake Spectra, vol. 29, no. 1, pp. 155-175, 2013.

[3] B. E. Tucker, "Reducing earthquake risk," Science, vol. 341, no. 6150, pp. 1070-1072, 2013.

[4] M. Erdik et al., "Earthquake risk assessment for Istanbul metropolitan area. Earthquake engineering and engineering vibration," vol. 2, no. 1, pp. 1-23, 2003.

[5] M. Wieland, M. Pittore, S. Parolai, J. Zschau, B. Moldobekov, and U. Begaliev, "Estimating building inventory for rapid seismic vulnerability assessment: Towards an integrated approach based on multisource imaging," Soil Dyn. Earthquake Eng., vol. 36, pp. 70-83, 2012.

[6] C. Geiß and H. Taubenböck, "Remote sensing contributing to assess earthquake risk: From a literature review towards a roadmap," Nat. Hazards, vol. 68, pp. 7-48, 2013.

[7] I. Riedel, P. Guéguen, F. Dunand, and S. Cottaz, "Macroscale vulnerability assessment of cities using association rule learning," Seismol. Res. Lett., vol. 85, no. 2, pp. 295-305, 2014.

[8] I. Riedel, P. Guéguen, M. Dalla Mura, E. Pathier, T. Leduc, and J. Chanussot, "Seismic vulnerability assessment of urban environments in moderate-to-low seismic hazard regions using association rule learning and support vector machine methods," Nat. Hazards, vol. 76, pp. 11111141, 2014, doi: 10.1007/s11069-014-1538-0.

[9] H. Taubenböck et al., "Assessing building vulnerability using synergistically remote sensing and civil engineering," in Urban and Regional Data Management, A. Kreck, M. Rumor, S. Zlatanova, and E. Fendel, Eds. New York, NY, USA: Taylor \& Francis, 2009, pp. 287-300.

[10] B. Borzi et al., "Vulnerability study on a large industrial area using satellite remotely sensed images," Bull. Earthquake Eng., vol. 9, pp. 675-690, 2011.

[11] G. Su et al., "An integrated method combining remote sensing data and local knowledge for the large-scale estimation of seismic loss risks to buildings in the context of rapid socioeconomic growth: A case study in Tangshan, China," Remote Sens., vol. 7, no. 3, pp. 2543-2601, 2015.

[12] F. Borfecchia et al., "Active and passive remote sensing for supporting the evaluation of the urban seismic vulnerability," Ital. J. Remote Sens., vol. 42, pp. 129-141, 2009.

[13] P. Matsuka, E. Pathier, P. Guéguen, and J. Chanussot, "Seismic vulnerability assessment using support vector machine classification for remote sensing and in-situ data," in Proc. 15th World Conf. Earthquake Eng., Lisbon, Portugal, Sep. 24-28, 2012 [Online]. Available: http://www.iitk.ac.in/nicee/wcee/article/WCEE2012_5147.pdf

[14] P. Matsuka, J. Chanussot, E. Pathier, and P. Guéguen, "A support vector regression approach for building seismic vulnerability assessment and evaluation from remote sensing and in-situ data," in Proc. IEEE Int. Geosci. Remote Sens. Symp., Munich, Germany, Jul. 22-27, 2012, pp. $7533-7536$.

[15] C. Geiß, H. Taubenböck, S. Tyagunov, A. Tisch, J. Post, and T. Lakes, "Assessment of seismic building vulnerability from space," Earthquake Spectra, vol. 30, no. 4, pp. 1553-1583, 2014.

[16] C. Geiß et al., "Estimation of seismic buildings structural types using multi-sensor remote sensing and machine learning techniques," ISPRS J. Photogramm. Remote Sens., vol. 104, pp. 175-188, 2015. 
[17] E. Banzhaf and R. Höfer, "Monitoring urban structure types as spatial indicators with CIR aerial photographs for a more effective urban environmental management," IEEE J. Sel. Topics Appl. Earth Observ. Remote Sens., vol. 1, no. 2, pp. 129-138, Jun. 2008.

[18] U. Heiden, W. Heldens, S. Roessner, K. Segl, T. Esch, and A. Mueller, "Urban structure type characterization using hyperspectral remote sensing and height information," Landsc. Urban Plann., vol. 105, pp. 361375, 2012.

[19] S. Pauleit and F. Duhme, "Assessing the environmental performance of land cover types for urban planning," Landscape Urban Plann., vol. 52, pp. $1-20,2000$.

[20] S. Niebergall, A. Loew, and W. Mauser, "Integrative assessment of informal settlements using VHR remote sensing data - The Delhi case study," IEEE J. Sel. Topics Appl. Earth Observ. Remote Sens., vol. 3, no. 1, pp. 193-205, Sep. 2008.

[21] M. Wurm, H. Taubenböck, A. Roth, and S. Dech, "Urban structuring using multisensoral remote sensing data: By the example of the German cities Cologne and Dresden," in Proc. Urban Remote Sens. Event, Shanghai, China, 2009, pp. 1-8.

[22] I. Baud, M. Kuffer, K. Pfeffer, R. Sliuzas, and S. Karuppannan, "Understanding heterogeneity in metropolitan India: The added value of remote sensing data for analyzing sub-standard residential areas," Int. J. Appl. Earth Observ. Geoinf., vol. 12, pp. 359-374, 2010.

[23] M. Bochow, K. Segl, and H. Kaufmann, "Automating the built-up process of feature-based fuzzy logic models for the identification of urban biotopes from hyperspectral remote sensing data," in Proc. Urban Remote Sens. Joint Event, Paris, France, 2007, pp. 1-8.

[24] W. Heldens, T. Esch, and U. Heiden, "Supporting urban micro climate modelling with airborne hyperspectral data," in Proc. IEEE Int. Geosci. Remote Sens. Symp., Munich, Germany, Jul. 23-27, 2012, pp. 15981601.

[25] B. Bechtel and C. Daneke, "Classification of local climate zones based on multiple earth observation data," IEEE J. Sel. Topics Appl. Earth Observ. Remote Sens., vol. 5, no. 4, pp. 1191-1202, Aug. 2012.

[26] M. Mueller, K. Segl, U. Heiden, and H. Kaufmann, "Potential of highresolution satellite data in the context of vulnerability of buildings," Nat. Hazards, vol. 38, pp. 247-258, 2006.

[27] M. Pittore and M. Wieland, "Towards a rapid probabilistic seismic vulnerability assessment using satellite and ground-based remote sensing," Nat. Hazards, vol. 68, pp. 115-145, 2013.

[28] R. Pal and K. Pal, "A review on image segmentation techniques," Pattern Recognit., vol. 26, no. 9, pp. 1277-1294, 1993.

[29] T. Blaschke, "Object based image analysis for remote sensing," ISPRS J. Photogramm. Remote Sens., vol. 65, pp. 2-16, 2010.

[30] C. H. Chen and P.-G. P. Ho, "Statistical pattern recognition in remote sensing," Pattern Recognit., vol. 41, no. 9, pp. 2731-2741, 2008.

[31] G. Camps-Valls, D. Tuia, L. Bruzzone, and J. A. Benediktsson, "Advances in hyperspectral image classification: Earth monitoring with statistical learning methods," IEEE Signal Process. Mag., vol. 31, no. 1, pp. 45-54, Jan. 2014.

[32] S. Steiniger, T. Lange, D. Burghardt, and R. Weibel, "An approach for the classification of urban building structures based on discriminant analysis techniques," Trans. GIS, vol. 12, no. 1, pp. 31-59, 2008.

[33] G. Tyc, J. Tulip, D. Schulten, M. Krischke, and M. Oxfort, "The RapidEye mission design," Acta Astronaut., vol. 56, pp. 213-219, 2005.

[34] G. Krieger et al., "TanDEM-X: A satellite formation for high-resolution SAR interferometry," IEEE Trans. Geosci. Remote Sens., vol. 45, no. 11, pp. 3317-3341, Nov. 2007.

[35] C. J. Tucker, "NASA's global orthorectified Landsat data set," Photogramm. Eng. Remote Sens., vol. 70, pp. 313-322, 2004.

[36] TurkStat. (2014). Turkish Statistical Institute [Online]. Available: http:// rapor.tuik.gov.tr/reports/rwservlet?adnksdb2\&ENVID=adnksdb2Env\& report=wa_buyukbelediye.RDF\&p_il1 $=34 \& p \_k o d=2 \& p \_y i l=2013 \& p$ dil $=1 \&$ desformat $=h t m l$, accessed on July 10, 2014.

[37] E. Cakti, "Issues with the earthquake vulnerability of Istanbul," Nat. Hazards, vol. 68, no. 1, pp. 227-228, 2013

[38] M. Erdik, Y. A. Biro, T. Onur, K. Sesetyan, and G. Birgoren, "Assessment of earthquake hazard in Turkey and neighboring regions," Ann. Geofis., vol. 42, no. 6, pp. 1125-1138, 1999.

[39] R. S. Stein, A. A. Barka, and J. H. Dieterich, "Progressive failure on the North Anatolian Fault since 1939 by earthquake stress triggering," Geophys. J. Int., vol. 128, pp. 594-604, 1997.

[40] T. Parsons, "Recalculated probability of $M \geq 7$ earthquakes beneath the Sea of Marmara, Turkey," J. Geophys. Res., vol. 109, B05304, 2004 [Online]. Available: https://profile.usgs.gov/myscience/upload_folder/ ci2010May25175655426802003JB002667.pdf
[41] J. Tigges, T. Lakes, and P. Hostert, "Urban vegetation classification: Benefits of multitemporal RapidEye satellite data," Remote Sens. Environ., vol. 136, pp. 66-75, 2013.

[42] M. Drusch et al., "Sentinel-2: ESA's optical high-resolution mission for GMES operational services," Remote Sens. Environ., vol. 120, pp. 25-36, 2012.

[43] J. R. Irons, J. L. Dwyer, and J. A. Barsi, "The next Landsat satellite: The Landsat data continuity mission," Remote Sens. Environ., vol. 122, pp. 11-21, 2012.

[44] H. Taubenböck, T. Esch, A. Felbier, M. Wiesner, A. Roth, and S. Dech, "Monitoring urbanization in mega cities from space," Remote Sens. Environ., vol. 117, pp. 162-176, 2012.

[45] S. A. Freeman, "Review of the development of the capacity spectrum method," J. Earthquake Technol., vol. 41, no. 1, pp. 1-13, 2004.

[46] FEMA, Rapid Visual Screening of Buildings for Potential Seismic Hazards. A Handbook, FEMA 154, 2nd ed. Redwood City, CA, USA: Applied Technology Council, 2002 [Online]. Available: http://www. unicef.org/education/files/VisualEvaluationFEMA_154.pdf

[47] R. Richter, Atmospheric/Topographic Correction for Satellite Imagery, ATCOR-2/3 User Guide, Version 7.0 BETA, 2008 [Online]. Available: http://www.dlr.de/eoc/Portaldata/60/Resources/dokumente/5_tech_mod/ atcor3_manual_2012.pdf

[48] T. Esch et al., "Urban footprint processor - Fully automated processing chain generating settlement masks from global data of the TanDEM-X mission," IEEE Geosci. Remote Sens. Lett., vol. 10, no. 6, pp. 1617-1621, Nov. 2013.

[49] R. M. Haralick, S. R. Sternberg, and X. Zhuang, "Image analysis using mathematical morphology," IEEE Trans. Pattern Anal. Mach. Intell., vol. 9, no. 4, pp. 532-550, Jul. 1987

[50] C. Geiß, M. Wurm, M. Breunig, A. Felbier, and H. Taubenböck, "Normalization of TanDEM-X DSM data in urban environments with morphological filters," IEEE Trans. Geosci. Remote Sens., vol. 53, no. 8, pp. 4348-4362, Aug. 2015.

[51] D. Shepard, "A two-dimensional interpolation function for irregularlyspaced data," in Proc. 23rd ACM Nat. Conf. (ACM'68), Aug. 27-29, 1968, pp. $517-524$

[52] M. Herold, X. Liu, and K. Clarke, "Spatial metrics and image texture for mapping urban land use," Photogramm. Eng. Remote Sens., vol. 69, pp. 991-1001, 2003.

[53] U. C. Benz, P. Hofmann, G. Willhauck, I. Lingenfelder, and M. Heynen, "Multi-resolution, object-oriented fuzzy analysis of remote sensing data for GIS-ready information," ISPRS J. Photogramm. Remote Sens., vol. 58, no. 3-4, pp. 239-258, 2004.

[54] T. Esch, M. Thiel, M. Bock, A. Roth, and S. Dech, "Improvement of image segmentation accuracy based on multiscale optimization procedure," IEEE Geosci. Remote Sens. Lett., vol. 5, no. 3, pp. 463-467, Jul. 2008

[55] G. M. Espindola, G. Camara, I. A. Reis, L. S. Bins, and A. M. Monteiro, "Parameter selection for region-growing image segmentation algorithms using spatial autocorrelation," Int. J. Remote Sens., vol. 27, no. 14, pp. 3035-3040, 2006.

[56] T. Hengl, "Finding the right pixel size," Comput. Geosci., vol. 32, pp. 1283-1298, 2006.

[57] T. R. Martha, N. Kerle, C. J. van Westen, V. Jetten, and K. V. Kumar, "Segment optimization and data-driven thresholding for knowledgebased landslide detection by object-based image analysis," IEEE Trans. Geosci. Remote Sens., vol. 49, no. 12, pp. 4928-4943, Dec. 2011.

[58] P. Lu, A. Stumpf, N. Kerle, and N. Casagli, "Object-oriented change detection for landslide rapid mapping," IEEE Geosci. Remote Sens. Lett., vol. 8, no. 4, pp. 701-705, Jul. 2011.

[59] R. Haralick, K. Shanmugam, and I. Dinstein, "Textural features for image classification," IEEE Trans. Syst. Man Cybern., vol. SMC-3, no. 6, pp. 610-621, Nov. 1973.

[60] F. Pacifici, M. Chini, and W. Emery, "A neural network approach using multi-scale textural metrics from very high resolution panchromatic imagery for urban land-use classification," Remote Sens. Environ., vol. 113, no. 6, pp. 1276-1292, 2009.

[61] J. W. Rouse, R. H. Haas, J. A. Schell, and D. W. Deering, "Monitoring vegetation systems in the great plains with ERTS," in Proc. 3rd Earth Resour. Technol. Satell.-1 Symp., 1974, pp. 309-317.

[62] L. Bruzzone and L. Carlin, "A multilevel context-based system for classification of very high spatial resolution images," IEEE Trans. Geosci. Remote Sens., vol. 44, no. 9, pp. 2587-2600, Sep. 2006.

[63] B. Johnson and Z. Xie, "Classifying a high resolution image of an urban area using super-object information," ISPRS J. Photogramm. Remote Sens., vol. 83, pp. 40-49, 2013. 
[64] G. F. Hughes, "On the mean accuracy of statistical pattern recognizers," IEEE Trans. Inf. Theory, vol. 14, no. 1, pp. 55-63, Jan. 1968.

[65] I. Kononenko, "Estimating attributes: Analysis and extensions of relief," in Proc. Eur. Conf. Mach. Learn., 1994, pp. 171-182.

[66] M. A. Hall, "Correlation-based feature selection for machine learning," Ph.D. dissertation, Dep. Comput. Sci., Univ. Waikato, Hamilton, New Zealand, 1999.

[67] M. Robnik-Šikonja and I. Kononenko, "Theoretical and empirical analysis of ReliefF and RReliefF," Mach. Learn. J., vol. 53, no. 1-2, pp. 23-69, 2003.

[68] V. N. Vapnik, The Nature of Statistical Learning Theory. New York, NY, USA: Springer, 1995.

[69] C. J. C. Burges, "A tutorial on support vector machines for pattern recognition," Data Mining Knowl. Discovery, vol. 2, pp. 121-167, 1998.

[70] P. Leinenkugel, T. Esch, and C. Künzer, "Settlement detection and impervious surface estimation in the Mekong Delta using optical and SAR remote sensing data," Remote Sens. Environ., vol. 115, pp. 3007-3019, 2011.

[71] M. Volpi, D. Tuia, F. Bovolo, M. Kanevski, and L. Bruzzone, "Supervised change detection in VHR images using contextual information and support vector machines," Int. J. Appl. Earth Observ. Geoinf., vol. 20, pp. 77-85, 2013.

[72] V. N. Vapnik, Statistical Learning Theory. Hoboken, NJ, USA: Wiley, 1998.

[73] C. C. Chang and C. J. Lin. (2001). LIBSVM: A Library for Support Vector Machines [Online]. Available: http://www.csie.ntu.edu.tw/ cjlin/libsvm, accessed on May 26, 2014

[74] B. Schölkopf and A. Smola, Learning with Kernels: Support Vector Machines, Regularization, Optimization, and Beyond. Cambridge, MA, USA: MIT Press, 2002.

[75] B. Schölkopf and A. J. Smola, "A tutorial on support vector regression," Statist. Comput., vol. 14, pp. 199-222, 2004.

[76] F. Melgani and L. Bruzzone, "Classification of hyperspectral remote sensing images with support vector machines," IEEE Trans. Geosci. Remote Sens., vol. 42, no. 8, pp. 1778-1790, Aug. 2004.

[77] G. Camps-Valls and L. Bruzzone, "Kernel-based methods for hyperspectral image classification," IEEE Trans. Geosci. Remote Sens., vol. 43, no. 6, pp. 1351-1362, Jun. 2005.

[78] G. Camps-Valls and L. Bruzzone, Kernel Methods for Remote Sensing Data Analysis. Hoboken, NJ, USA: Wiley, 2009.

[79] G. Mountrakis, J. Im, and C. Ogole, "Support vector machines in remote sensing: A review," ISPRS J. Photogramm. Remote Sens., vol. 66, pp. 247-259, 2011

[80] S. Salcedo-Sanz, J. L. Rojo-Alvarez, M. Martinez-Ramon, and G. CampsValls, "Support vector machines in engineering: An overview," Wiley Interdiscip. Rev. Data Mining Knowl. Discovery, vol. 4, no. 3, pp. 234267, 2014.

[81] J. Muñoz-Marí, F. Bovolo, L. Gomez-Chova, L. Bruzzone, and G. CampValls, "Semisupervised one-class support vector machines for classification of remote sensing data," IEEE Trans. Geosci. Remote Sens., vol. 48, no. 8, pp. 3188-3197, Aug. 2010.

[82] M. Marconcini, D. Fernandez-Prieto, and T. Buchholz, "Targeted landcover classification," IEEE Trans. Geosci. Remote Sens., vol. 52, no. 7, pp. 4173-4193, Jul. 2014.

[83] G. M. Foody, "Thematic map comparison: Evaluating the statistical significance of differences in classification accuracy," Photogramm. Eng. Remote Sens., vol. 70, pp. 627-633, 2004.

[84] C. W. Hsu and C. J. Lin, "A comparison of methods for multi-class support vector machines," IEEE Trans. Neural Netw., vol. 13, no. 2, pp. 415-425, Mar. 2002.

[85] C. W. Hsu, C. C. Chang, and C. J. Lin, "A practical guide to support vector classification," Dep. Comput. Sci., Nat. Taiwan Univ., Tech. Rep., 2010 [Online]. Available: http://www.csie.ntu.edu.tw/ cjlin/ papers/guide/guide.pdf

[86] L. Bruzzone, M. Chi, and M. Marconcini, "A novel transductive SVM for semisupervised classification of remote-sensing images," IEEE Trans. Geosci. Remote Sens., vol. 44, no. 11, pp. 3363-3373, Nov. 2006.

[87] D. Tuia, F. Ratle, F. Pacifici, M. F. Kanevski, and W. J. Emery, "Active learning methods for remote sensing image classification," IEEE Trans. Geosci. Remote Sens., vol. 47, no. 7, pp. 2218-2232, Jul. 2009.

[88] D. Tuia, L. Copa, M. Kanevski, and J. Munoz-Mari, "A survey of active learning algorithms for supervised remote sensing image classification," IEEE J. Sel. Topics Signal Process., vol. 5, no. 3, pp. 606-617, Jun. 2011.
[89] A. Stumpf, N. Lachiche, J. P. Malet, N. Kerle, and A. Puissant, "Active learning in the spatial domain for remote sensing image classification," IEEE Trans. Geosci. Remote Sens., vol. 52, no. 5, pp. 2492-2507, May 2014.

[90] E. Pasolli, F. Melgani, D. Tuia, F. Pacifici, and W. J. Emery, "SVM active learning approach for image classification using spatial information," IEEE Trans. Geosci. Remote Sens., vol. 52, no. 4, pp. 2217-2233, Apr. 2014

[91] B. Demir, L. Minello, and L. Bruzzone, "Definition of effective training sets for supervised classification of remote sensing images by a novel cost-sensitive active learning method," IEEE Trans. Geosci. Remote Sens., vol. 52, no. 2, pp. 1272-1284, Feb. 2014

[92] D. Tuia, E. Pasolli, and W. J. Emery, "Using active learning to adapt remote sensing image classifiers," Remote Sens. Environ., vol. 115, pp. 2232-2242, 2011.

[93] F. Löw, P. Knöfel, and C. Conrad, "Analysis of uncertainty in multitemporal object-based classification," ISPRS J. Photogramm. Remote Sens., vol. 105, pp. 91-106, 2015.

[94] M. Pittore, "Focus maps: A means of prioritizing data collection for efficient geo-risk management," Ann. Geophys., vol. 58, no. 1, S0107, 2015, doi: $10.4401 /$ ag-6692.

[95] B. Krawczyk, M. Wozniak, and B. Cyganek, "Clustering-based ensembles for one-class classification," Inf. Sci., vol. 264, pp. 182-195, 2014.

[96] C. Desir, S. Bernard, C. Petitjean, and L. Heutte, "One class random forests," Pattern Recognit., vol. 46, no. 12, pp. 3490-3506, 2013.

[97] C. E. Rasmussen and C. K. I. Williams, Gaussian Processes for Machine Learning. Cambridge, MA, USA: MIT Press, 2006.

[98] J. Verrelst et al., "Machine learning regression algorithms for biophysical parameter retrieval: Opportunities for Sentinel-2 and -3," Remote Sens. Environ., vol. 118, pp. 127-139, 2012.

[99] M. Wieland and M. Pittore, "Performance evaluation of machine learning algorithms for urban pattern recognition from multi-spectral satellite images," Remote Sens., vol. 6, pp. 2912-2939, 2014.

[100] C. Cortes and V. Vapnik, "Support vector networks," Mach. Learn., vol. 20, pp. 273-297, 1995.

[101] B. Schölkopf, R. C. Williamson, A. Smola, and J. Shawe-Taylor, "Support vector method for novelty detection," in Proc. Adv. Neural Inf. Process. Syst., Denver, CO, USA, 1999, vol. 12, pp. 582-588.

[102] B. Schölkopf, J. C. Platt, J. Shawe-Taylor, A. J. Smola, and R. C. Williamson, "Estimating the support of a high dimensional distribution," Neural Comput., vol. 13, no. 7, pp. 1443-1471, 2001.

[103] H. Drucker, C. J. C. Burges, L. Kaufman, A. J. Smola, and V. Vapnik, "Support vector regression machines," Adv. Neural Inf. Process. Syst., vol. 9, pp. 155-161, 1997.

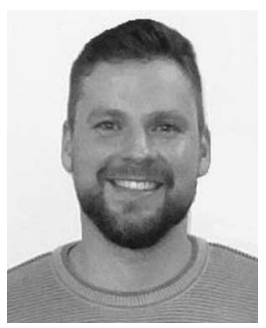

Christian Geiß (M'15) received the M.Sc. degree in applied geoinformatics from the Paris Lodron University of Salzburg, Salzburg, Austria, in 2010, and the Ph.D. degree (Dr.rer.nat.) from Humboldt University of Berlin, Berlin, Germany, in 2014.

Since 2010, he has been with the German Remote Sensing Data Center (DFD), German Aerospace Center (DLR), Munich, Germany. His research interests include renewable energy, earthquake risk assessment, methods for remote sensing data processing, techniques of multimodal remote sensing, machine learning, and pattern recognition, specifically applied to the field of natural hazard risk research.

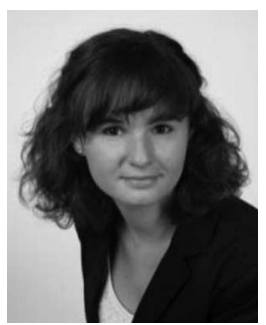

Marianne Jilge was born in Munich, Germany, in 1988. She received the B.Eng. degree in cartography and geomedia technology from the University of Applied Sciences, Munich, Germany, in 2012, and the M.Sc. degree in applied geoinformatics from the Paris Lodron University of Salzburg, Salzburg, Austria, in 2014. Currently, she is pursuing the Ph.D. degree at the Ruhr University of Bochum, Bochum, Germany.

Her research interests include analysis of urban areas with the prospective spaceborne hyperspectral satellite mission EnMAP in cooperation with the German Aerospace Agency (DLR) 


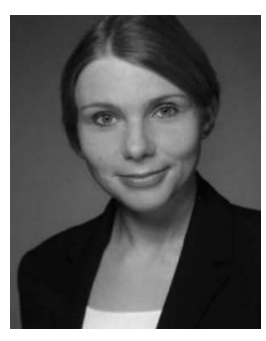

Tobia Lakes studied geography at the University of Bonn, Bonn, Germany, the University of Duisburg, Essen, Germany, and the University of Belfast, Belfast, U.K. She received the Diploma degree in geography from the University of Bonn, in 2001, and the Ph.D. degree (Dr.-Ing.) from the Technische Universität Berlin, Berlin, Germany, in 2006.

Since 2007, she has been an Assistant Professor in geoinformatics with the Geography Department, Humboldt-Universität zu Berlin, Berlin, Germany. Her research interests include the integration of remote-sensing data with additional data to model the human-environment system in a spatially explicit way, urban areas, and land use change.

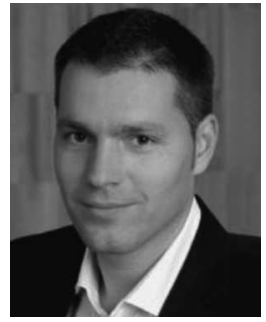

Hannes Taubenböck received the Diploma degree in geography from Ludwig-Maximilians Universität München, Munich, Germany, in 2004, and the $\mathrm{Ph} . \mathrm{D}$. degree (Dr.rer.nat.) in geography from JuliusMaximilian's University of Würzburg, Würzburg, Germany, in 2008.

In 2005, he joined the German Remote Sensing Data Center (DFD), German Aerospace Center (DLR), Munich, Germany, working on the topic "Mega cities and Natural Disasters." He is a Lecturer with the University of Würzburg. In 2010, he was a Scientific Employee with DLR-DFD and became the Head of the "Modelling and geostatistical methods" Team in 2013. His research activities as Postdoc with the University of Würzburg (2007-2010) concentrated on the development of algorithms for information extraction from multisensoral remotely sensed data and subsequent value adding for transforming scientific results into practical value. 